TRANSACTIONS OF THE

AMERICAN MATHEMATICAL SOCIETY

Volume 359, Number 6, June 2007, Pages 2597-2623

S 0002-9947(07)03979-7

Article electronically published on January 25, 2007

\title{
PROJECTIVITY AND FREENESS OVER COMODULE ALGEBRAS
}

\author{
SERGE SKRYABIN
}

\begin{abstract}
Let $H$ be a Hopf algebra and $A$ an $H$-simple right $H$-comodule algebra. It is shown that under certain hypotheses every $(H, A)$-Hopf module is either projective or free as an $A$-module and $A$ is either a quasi-Frobenius or a semisimple ring. As an application it is proved that every weakly finite (in particular, every finite dimensional) Hopf algebra is free both as a left and a right module over its finite dimensional right coideal subalgebras, and the latter are Frobenius algebras. Similar results are obtained for $H$-simple $H$-module algebras.
\end{abstract}

\section{INTRODUCTION}

Let $H$ be a Hopf algebra over a field $k$. Starting from the work of Radford [31, 32 the question about the freeness and projectivity of $H$ over its Hopf subalgebras aroused a substantial amount of interest. Besides the pointed case studied in 31. it is known that $H$ is a projective module over any Hopf subalgebra whenever $H$ is commutative [41, Cor. 1]. Takeuchi's original proof of this fact was based on the faithful flatness as a preliminary step. By itself the faithful flatness of $H$ over Hopf subalgebras is not so straightforward. When the subalgebra is reduced one can apply the theorem on generic flatness; in general one has to make a reduction to that case.

The first contribution to the theory that I am going to propose consists of showing that the projectivity result just mentioned can be derived directly from a known projectivity criterion in terms of the Fitting invariants. The argument is very short and applies actually in a more general situation. Suppose that $G$ is any covariant functor from the category of commutative $k$-algebras to the category of groups. It makes sense to say, what does it mean for $G$ to operate on a commutative algebra $A$ by automorphisms [5]. If such an action is given one can introduce the notion of $A, G$-modules. These are $A$-modules equipped with a compatible $G$ module structure. It is shown in Proposition 1.1 that all Fitting invariants of an $A$-finite $A, G$-module $M$ are $G$-stable ideals of $A$. If $A$ is $G$-simple in the sense that $A$ has no nontrivial $G$-stable ideals, this immediately implies the projectivity of $M$ as an $A$-module (Corollary 1.3 to Theorem 1.2). This gives a better result

Received by the editors February 27, 2004 and, in revised form, February 11, 2005.

2000 Mathematics Subject Classification. Primary 16W30.

This research was supported by the project "Construction and applications of non-commutative geometry" from FWO Vlaanderen. I would like to thank the Free University of Brussels VUB for their hospitality during the time the work was conducted.

(C)2007 American Mathematical Society Reverts to public domain 28 years from publication 2597 
even in the case of an algebraic group $G$ operating rationally on $A$. Doraiswamy 9] treated connected algebraic groups $G$ and finitely generated algebras $A$, but the method there was based on the conclusion that $A$ is an integral domain under the hypotheses stated. There are two cases where our result can be translated into the language of Hopf algebras (Corollaries 1.5, 1.6). One can take $G$ to be either a group scheme or a formal group scheme, and such group functors correspond, respectively, to commutative and to cocommutative Hopf algebras. In the first of these cases one easily obtains an improvement by removing the finiteness condition on modules.

A generalization to noncommutative Hopf algebras presents serious difficulty. The celebrated achievement of Nichols and Zoeller consisted in proving that every finite dimensional Hopf algebra $H$ is a free module over its Hopf subalgebras [27. In [18. Masuoka extended this result by showing that $H$ is free both as a left and a right module over its right coideal subalgebra $A$ if and only if $A$ is Frobenius. Several other conditions equivalent to $A$ being Frobenius were given in [13, [18, [21. The notion of coideal subalgebras appears to be of fundamental importance. According to [18 there is a bijective correspondence between the Frobenius right coideal subalgebras in $H$ and in $H^{*}$. Whether all coideal subalgebras of $H$ are Frobenius was known to be true under the assumption that $H$ has a cocommutative coradical [17] and under the assumption that $H$ is involutory and char $k$ is either 0 or $>\operatorname{dim} H$ [18. The primary motivation behind the present article was to solve the last question for every finite dimensional $H$. Our approach also provides a new proof of Nichols and Zoeller's theorem.

Again it is natural to work in more general settings. Let $H$ be an arbitrary Hopf algebra over a field $k$ and $A$ a right $H$-comodule algebra. There are categories of Hopf modules $\mathcal{M}_{A}^{H}$ and ${ }_{A} \mathcal{M}^{H}$ introduced by Doi (e.g., [6, [7, 8] ) as a generalization of their special cases due to Takeuchi [39], 41]. An $(H, A)$-Hopf module is either right or left $A$-module equipped with a compatible $H$-comodule structure. We say that $A$ is $H$-simple if $A$ has no nontrivial $H$-costable (two-sided) ideals. Theorem 3.5 states that all objects of $\mathcal{M}_{A}^{H}$ are projective $A$-modules provided that $A$ is $H$-simple, semilocal and satisfies one technical condition $(\mathrm{C})$ concerned with the weak finiteness of certain rings. The last condition is not restrictive for many applications as, for instance, left or right Noetherian rings, as well as rings finitely generated as modules over commutative subrings are always weakly finite. Condition (C) is used to check that certain ideals of $A$ are $H$-costable. If $\operatorname{dim} A<\infty$, in addition to previous hypotheses, then we will see in Theorem 4.2 that $A$ is Frobenius and all objects of both $\mathcal{M}_{A}^{H}$ and ${ }_{A} \mathcal{M}^{H}$ are projective $A$-modules. Theorem 4.5 ensures that $A$ is a quasi-Frobenius ring under weaker assumptions about $A$. If $\operatorname{dim} H<\infty$, then the hypotheses can be further weakened, and we also show in Theorem 5.2 that $A$ is semisimple provided that so is $H$.

In order to apply the previous results to coideal subalgebras one has to know that they are $H$-simple. This is based on Proposition 3.7: a right Artinian $H$-comodule algebra $A$ satisfying (C) is $H$-simple provided that there exists a maximal ideal of $A$ containing no nonzero $H$-costable ideals. Suppose next that $A$ is a finite dimensional right coideal subalgebra of $H$. Under the assumption that $H$ is weakly finite the conclusions of Theorem 4.2 hold true for $A$. Thus $A$ is Frobenius not only for finite dimensional $H$ but also under much weaker assumptions. Moreover, all objects of both $\mathcal{M}_{A}^{H}$ and ${ }_{A} \mathcal{M}^{H}$ are free $A$-modules, and the two categories are equivalent to 
the categories of comodules over certain quotient coalgebras of $H$. In addition the so-called normal basis property is fulfilled for $H$. All this is stated in Theorem 6.1.

It is worth noting that the finiteness assumptions in the last result are necessary. Nichols and Zoeller 28 gave an example of a Hopf algebra $H$ containing a twodimensional Hopf subalgebra $A$ such that there exists a finite dimensional object of ${ }_{A} \mathcal{M}^{H}$ which is not a free $A$-module. We recall this example in section 6 and show directly that $H$ is not weakly finite here. On the other hand, the infinite dimensional coideal subalgebras are not always $H$-simple and the projectivity over them may fail even when $H$ is commutative. As an example take $H$ to be the group algebra of the free cyclic group, say with a generator $g$, and $A$ the subalgebra of $H$ generated by $g$. At least two related results were established in the literature without finiteness restrictions on $H$, however. If $A$ is a finite dimensional Hopf subalgebra of any $H$ and $A$ is either semisimple or normal in $H$, then $H$ is a free A-module [29], 36].

In section 7 of the paper we dualize the projectivity result to the case of semilocal $H$-module algebras. In conclusion I would like to thank A. Masuoka and S. Montgomery for helpful comments.

\section{NOTATIONS AND CONVENTIONS}

Let $k$ be the ground field. All algebras and coalgebras are over $k$, and $k$ serves normally as the base ring for functors $\otimes$ and Hom. If $A$ is an algebra and $D$ a coalgebra, denote by ${ }_{A} \mathcal{M}, \mathcal{M}_{A},{ }^{D} \mathcal{M}, \mathcal{M}^{D}$ the categories of left $A$-modules, right $A$-modules, left $D$-comodules and right $D$-comodules, respectively. Objects of the category $D_{\mathcal{M}}$ are vector spaces equipped with a pair of commuting structures of a right $A$-module and a left $D$-comodule so that all elements of $A$ operate as $D$-comodule endomorphisms. The category ${ }_{A}^{D} \mathcal{M}$ is defined similarly using left $A$ modules.

Let $H$ be a Hopf algebra with the comultiplication $\Delta$, the counit $\varepsilon$ and the antipode $s$. Either [25] or [38] can be used as a general reference on Hopf algebras. A right $H$-comodule algebra is an algebra $A$ together with a right $H$-comodule structure $\rho_{A}: A \rightarrow A \otimes H$ such that $\rho_{A}$ is a homomorphism of (unital) algebras. A left $H$ module algebra is an algebra $A$ together with a left $H$-module structure such that the map $\tau_{A}: A \rightarrow \operatorname{Hom}(H, A)$ defined by the rule $\tau_{A}(a)(h)=h a$ for $a \in A$ and $h \in H$ is an algebra homomorphism. Here $\operatorname{Hom}(H, A)$ is regarded as an algebra with respect to the convolution multiplication. We omit the prefix "right" for comodule algebras and the prefix "left" for module algebras. The subalgebra of invariants of an $H$-comodule algebra $A$ is defined to be

$$
A^{H}=\{a \in A \mid \rho(a)=a \otimes 1\} .
$$

With each comodule algebra $A$ one associates the categories $\mathcal{M}_{A}^{H}$ and ${ }_{A} \mathcal{M}^{H}$. Their objects are either right or left $A$-modules together with a compatible right $H$-comodule structure. For each module algebra $A$ we denote by ${ }_{H} \mathcal{M}_{A}$ the category whose objects are right $A$-modules together with a compatible left $H$-module structure. The compatibility condition in each of the respective cases is as follows:

$$
\begin{gathered}
\rho_{M}(m a)=\rho_{M}(m) \rho_{A}(a), \quad \rho_{M}(a m)=\rho_{A}(a) \rho_{M}(m), \\
\tau_{M}(m a)=\tau_{M}(m) \tau_{A}(a)
\end{gathered}
$$

where $m \in M$ and $a \in A$. Here $\rho_{M}: M \rightarrow M \otimes H$ is the comodule structure map, and we regard $M \otimes H$ as either right or left $A \otimes H$-module letting $A$ operate on 
the first tensorand and $H$ on the second tensorand via multiplications. The map $\tau_{M}: M \rightarrow \operatorname{Hom}(H, M)$ is defined by the rule $\tau_{M}(m)(h)=h m$ for $m \in M$ and $h \in H$, and we regard $\operatorname{Hom}(H, M)$ as a right $\operatorname{Hom}(H, A)$-module with respect to the convolution action. Given linear functions $\xi: H \rightarrow M$ and $\eta: H \rightarrow A$, one obtains $\xi \eta$ as the composite

$$
H \stackrel{\Delta}{\longrightarrow} H \otimes H \stackrel{\xi \otimes \eta}{\longrightarrow} M \otimes A \longrightarrow M .
$$

We often omit the subscripts in the notations $\rho_{A}, \rho_{M}, \tau_{A}, \tau_{M}$. Another way to express the compatibility of module and comodule structures in the categories above is to say that the module structure map $M \otimes A \rightarrow M$ or $A \otimes M \rightarrow M$ is a morphism in either $\mathcal{M}^{H}$ or ${ }_{H} \mathcal{M}$. Here we use the tensor product of two comodule or two module structures defined in [25, §1.8]. When necessary, we regard $k$ as a trivial $H$-module or $H$-comodule.

Objects of ${ }_{H} \mathcal{M}_{A}$ can be identified with the left modules over the smash product algebra $A^{\mathrm{op}} \# H^{\mathrm{cop}}$. Here $A^{\mathrm{op}}$ is $A$ taken with the opposite multiplication and $H^{\mathrm{cop}}$ is $H$ taken with the opposite comultiplication and the same multiplication. If $A$ is commutative and $H$ cocommutative, then ${ }_{H} \mathcal{M}_{A} \approx{ }_{A \# H} \mathcal{M}$.

An object $M$ of $\mathcal{M}_{A}^{H},{ }_{A} \mathcal{M}^{H}$, or ${ }_{H} \mathcal{M}_{A}$ will be called $A$-finite if it is finitely generated as an $A$-module. We say that $M$ is locally $A$-finite if it coincides with the union of its $A$-finite subobjects. The same terminology will be used in the category ${ }_{A, G} \mathcal{M}$ introduced in section 1. Every object $M$ of either $\mathcal{M}_{A}^{H}$ or ${ }_{A} \mathcal{M}^{H}$ is locally $A$ finite. Indeed, the $A$-modules generated by the finite dimensional $H$-subcomodules of $M$ are $A$-finite subobjects of $M$.

An "ideal" will be understood as a two-sided ideal unless explicitly specified otherwise. The ideals in a (co)module algebra $A$ which are respected by the (co)module structure will be termed $H$-(co)stable. The algebra $A$ will be called $H$-simple if it has no nonzero proper $H$-(co)stable ideals. Denote by $\operatorname{Max} R$ the set of all maximal ideals in a ring $R$.

Note that $H$ is an $H$-comodule algebra with respect to $\Delta$ and $H$ is a simple object of $\mathcal{M}_{H}^{H}$. By [38, Th. 4.1.1] every $M \in \mathcal{M}_{H}^{H}$ decomposes as $M_{0} \otimes H$ where $M_{0}=\{m \in M \mid \rho(m)=m \otimes 1\}$. For $M=H$ one has $M_{0}=k$ so that there is no room for nontrivial subobjects.

We use Sweedler's symbolic notations for comultiplication. If $h \in H$ and $m \in M$ where $M \in \mathcal{M}^{H}$, then

$$
\begin{array}{cc}
\Delta(h)=\sum_{(h)} h_{(1)} \otimes h_{(2)}, & (\Delta \otimes \mathrm{id}) \circ \Delta(h)=\sum_{(h)} h_{(1)} \otimes h_{(2)} \otimes h_{(3)}, \\
\rho(m)=\sum_{(m)} m_{(0)} \otimes m_{(1)}, & (\rho \otimes \mathrm{id}) \circ \rho(m)=\sum_{(m)} m_{(0)} \otimes m_{(1)} \otimes m_{(2)} .
\end{array}
$$

\section{Fully commutative case}

Suppose that $A$ is a commutative ring and $M$ a finitely generated $A$-module. For each $i \geq 0$ the $i$ th Fitting invariant $\operatorname{Fitt}_{i}(M)$ of $M$ is defined as follows. Taking an epimorphism of $A$-modules $\pi: F \rightarrow M$ where $F$ is a free $A$-module, say of rank $n$, one sets $\operatorname{Fitt}_{i}(M)=A$ when $i \geq n$; otherwise $\operatorname{Fitt}_{i}(M)$ is the ideal of $A$ generated by the determinants of all $(n-i) \times(n-i)$ matrices $\left[f_{j}\left(x_{l}\right)\right]_{1 \leq j, l \leq n-i}$ where $f_{1}, \ldots, f_{n-i}$ run through $\operatorname{Hom}_{A}(F, A)$ and $x_{1}, \ldots, x_{n-i}$ run through $\operatorname{Ker} \pi$. It is well known that this definition does not depend on the choice of a presentation 
of $M$. We formally put Fitt $-1(M)=0$. We will need two properties of the Fitting ideals (see [10, Cor. 20.5 and Prop. 20.8]):

(F1) $\operatorname{Fitt}_{i}\left(B \otimes_{A} M\right)=\operatorname{Fitt}_{i}(M) B$ whenever $B$ is a commutative $A$-algebra.

(F2) For $M$ to be a projective $A$-module of constant rank $r \geq 0$ it is necessary and sufficient that $\operatorname{Fitt}_{r}(M)=A$ and $\operatorname{Fitt}_{r-1}(M)=0$.

Denote by $\mathrm{Comm}_{k}$ the category of commutative $k$-algebras. A group $k$-functor $G$ is any functor from $\mathrm{Comm}_{k}$ to the category of groups [5]. Thus $G$ associates a group $G(R)$ with each $R \in C^{\circ} m_{k}$ and a group homomorphism $G(\varphi): G(R) \rightarrow G\left(R^{\prime}\right)$ with each homomorphism of commutative algebras $\varphi: R \rightarrow R^{\prime}$. A $G$-module is a vector space $V$ together with $R$-linear actions of the groups $G(R)$ on the $R$-modules $V \otimes R$ which are given for each $R \in C o m m_{k}$ and are compatible with morphisms in $C$ omm $_{k}$, that is, whenever $g \in G(R)$ and $\varphi: R \rightarrow R^{\prime}$ is an algebra homomorphism, the transformation of $V \otimes R^{\prime}$ afforded by $G(\varphi)(g) \in G\left(R^{\prime}\right)$ is the $R^{\prime}$-linear extension of the transformation of $V \otimes R$ afforded by $g$ [5, Ch. II, §2].

Assume further that $A \in C o m m_{k}$. One says that a group $k$-functor $G$ operates on $A$ by automorphisms if $A$ is given a $G$-module structure and for each $R \in C o m m_{k}$ the group $G(R)$ acts on $A \otimes R$ as a group of algebra automorphisms. If such an action is given, an ideal $I$ of $A$ will be called $G$-stable if $I \otimes R$ is stable under $G(R)$ for each $R \in C \mathrm{Cmm}_{k}$. The definition of $G$-stable subalgebras is similar. We say that $A$ is $G$-simple if $A$ has no nonzero proper $G$-stable ideals.

An $A, G$-module $M$ is an $A$-module together with a $G$-module structure which satisfies the following compatibility condition:

$$
g(a m)=(g a)(g m) \quad \text { for all } g \in G(R), a \in A \otimes R, m \in M \otimes R .
$$

Denote by $A, G \mathcal{M}$ the category of all $A, G$-modules.

If $G$ is a group scheme, then the notion of $A, G$-modules can be interpreted geometrically in terms of $G$-linearized quasicoherent sheaves on the affine scheme Spec $A$ [26, Ch. I, §3]. Such structures have also been studied in purely algebraic context. For instance, if $\Gamma$ is an ordinary group and $G$ the constant group functor such that $G(R)=\Gamma$ for each $R \in C$ omm $_{k}$ and $G(\varphi)$ is the identity map $\Gamma \rightarrow \Gamma$ for each morphism $\varphi$ in $C o m m_{k}$, an $A, G$-module is just a module over the skew group ring $A * \Gamma$.

Proposition 1.1. If $M \in{ }_{A, G} \mathcal{M}$ is $A$-finite, then all its Fitting ideals $\operatorname{Fitt}_{i}(M)$ are $G$-stable.

Proof. Suppose that $g \in G(R)$ where $R \in C o m m_{k}$. For every $A \otimes R$-module $N$ one can define a new $A \otimes R$-module ${ }^{g} N$ such that ${ }^{g} N=N$ as abelian groups and each $a \in A \otimes R$ operates in ${ }^{g} N$ as $g^{-1}(a)$ does in $N$. We claim that

$$
\operatorname{Fitt}_{i}\left({ }^{g} N\right)=g\left(\operatorname{Fitt}_{i}(N)\right) \text {. }
$$

To prove this equality consider an epimorphism of $A \otimes R$-modules $\pi: F \rightarrow N$ where $F$ is a free $A \otimes R$-module, say of rank $n$. The same map $\pi$ is also an epimorphism of $A \otimes R$-modules ${ }^{g} F \rightarrow{ }^{g} N$. Clearly ${ }^{g} F$ is a free $A \otimes R$-module of rank $n$. Indeed, any basis for $F$ is also a basis for ${ }^{g} F$. In particular, both sides of $(* *)$ are equal to $A \otimes R$ when $i \geq n$. Assume that $0 \leq i<n$. There is a bijection

$$
\operatorname{Hom}_{A \otimes R}(F, A \otimes R) \rightarrow \operatorname{Hom}_{A \otimes R}\left({ }^{g} F, A \otimes R\right)
$$


given by $f \mapsto{ }^{g} f$ where $\left({ }^{g} f\right)(x)=g(f(x))$ for $x \in F$. As $g$ operates on $A \otimes R$ as an algebra automorphism, we have

$$
\operatorname{det}\left[\left({ }^{g} f_{j}\right)\left(x_{l}\right)\right]_{1 \leq j, l \leq n-i}=g\left(\operatorname{det}\left[f_{j}\left(x_{l}\right)\right]_{1 \leq j, l \leq n-i}\right)
$$

whenever $f_{1}, \ldots, f_{n-i} \in \operatorname{Hom}_{A \otimes R}(F, A \otimes R)$ and $x_{1}, \ldots, x_{n-i} \in \operatorname{Ker} \pi$. The determinants on the left and right hand sides of this formula generate the two ideals in $(* *)$, whence the claim.

We will apply $(* *)$ to the $A \otimes R$-module $N=M \otimes R$. First of all, ${ }^{g} N \cong N$ in this case because the transformation of $N$ afforded by $g$ is an isomorphism between the two $A \otimes R$-module structures according to $(*)$. Hence $\operatorname{Fitt}_{i}\left({ }^{g} N\right)=\operatorname{Fitt}_{i}(N)$ so that (**) just says that $\operatorname{Fitt}_{i}(N)$ is stable under $g$. Property (F1) applied to the $A$-algebra $B=A \otimes R$ shows, however, that $\operatorname{Fitt}_{i}(N)=\operatorname{Fitt}_{i}(M) \otimes R$.

Theorem 1.2. Suppose that $B$ is a commutative $A$-algebra such that $I B=B$ for every $G$-stable ideal $I \neq 0$ of $A$. If $M \in{ }_{A, G} \mathcal{M}$ is locally $A$-finite, then $B \otimes_{A} M$ is a projective B-module. The latter has constant rank whenever $M$ is A-finite.

Proof. Suppose first that $M$ is $A$-finite. Since $\operatorname{Fitt}_{i}(M)$ is a $G$-stable ideal of $A$ by Proposition 1.1, it follows from (F1) that $\operatorname{Fitt}_{i}\left(B \otimes_{A} M\right)$ is either 0 or $B$ for each $i \geq-1$. On the other hand, the latter ideal is 0 for $i=-1$ and is $B$ for sufficiently large $i$. Then there exists $r \geq 0$ such that $\operatorname{Fitt}_{i}\left(B \otimes_{A} M\right)$ is $B$ for $i=r$ and is 0 for $i=r-1$. By (F2) the $B$-module $B \otimes_{A} M$ is projective of rank $r$.

Now consider the general case. Denote by $\mathcal{G}$ and $\mathcal{F}$ the sets whose elements are all $A, G$-submodules of $M$ and the $A$-finite ones, respectively. Given $N, N^{\prime} \in \mathcal{G}$ such that $N \subset N^{\prime}$, put

$$
T_{N N^{\prime}}=\operatorname{Im}\left(B \otimes_{A} N \stackrel{\text { can }}{\longrightarrow} B \otimes_{A} N^{\prime}\right) .
$$

Step 1. Also put $T_{N}=T_{N M}$ for short. Thus $T_{N} \subset B \otimes_{A} M$ is a $B$-submodule. We will show that $T_{N}$ is a projective $B$-module whenever $N \in \mathcal{F}$. The set $\mathcal{F}$ is directed by inclusion and $M=\bigcup_{N^{\prime} \in \mathcal{F}} N^{\prime}$ by local finiteness of $M$. Since tensor products commute with direct limits, we have $B \otimes_{A} M \cong \lim _{N^{\prime} \in \mathcal{F}} B \otimes_{A} N^{\prime}$ and

$$
T_{N} \cong \lim _{N^{\prime} \in \mathcal{F}_{N}} T_{N N^{\prime}}
$$

where $\mathcal{F}_{N}=\left\{N^{\prime} \in \mathcal{F} \mid N \subset N^{\prime}\right\}$. If $N^{\prime} \in \mathcal{F}_{N}$, then both $N^{\prime}$ and $N^{\prime} / N$ are $A$-finite objects of ${ }_{A, G} \mathcal{M}$, whence $B \otimes_{A} N^{\prime}$ and $B \otimes_{A} N^{\prime} / N$ are projective $B$ modules of constant rank. Since $\left(B \otimes_{A} N^{\prime}\right) / T_{N N^{\prime}} \cong B \otimes_{A} N^{\prime} / N$ by right exactness of tensor products, we deduce that $T_{N N^{\prime}}$ is a projective $B$-module of constant rank. Suppose that $N^{\prime}, N^{\prime \prime} \in \mathcal{F}_{N}$ and $N^{\prime} \subset N^{\prime \prime}$. Then the canonical morphism $B \otimes_{A} N^{\prime} \rightarrow B \otimes_{A} N^{\prime \prime}$ in ${ }_{B} \mathcal{M}$ maps $T_{N N^{\prime}}$ onto $T_{N N^{\prime \prime}}$. The kernel, say $K$, of the induced epimorphism $T_{N N^{\prime}} \rightarrow T_{N N^{\prime \prime}}$ is a projective $B$-module of constant rank equal to $\operatorname{rk} T_{N N^{\prime}}-\operatorname{rk} T_{N N^{\prime \prime}}$. We see that $\operatorname{rk} T_{N N^{\prime}} \geq \operatorname{rk} T_{N N^{\prime \prime}}$ and one has an equality here if and only if $K=0$. Pick $N^{\prime} \in \mathcal{F}_{N}$ for which $\operatorname{rk} T_{N N^{\prime}}$ attains the minimum value. Then $T_{N N^{\prime}}$ is mapped isomorphically onto $T_{N N^{\prime \prime}}$ for each $N^{\prime \prime} \in \mathcal{F}_{N}$ such that $N^{\prime} \subset N^{\prime \prime}$, and it follows that $T_{N} \cong T_{N N^{\prime}}$.

Step 2. Next we will prove that $T_{N^{\prime}} / T_{N}$ is a projective $B$-module whenever $N, N^{\prime} \in \mathcal{G}$ are such that $N \subset N^{\prime}$ and $N^{\prime} / N$ is $A$-finite. Since tensor products are right exact, we have $\left(B \otimes_{A} M\right) / T_{N} \cong B \otimes_{A} M / N$. This induces an isomorphism of $T_{N^{\prime}} / T_{N}$ onto the image of the canonical map $B \otimes_{A} N^{\prime} / N \rightarrow B \otimes_{A} M / N$. It remains to apply Step 1 to the locally $A$-finite object $M / N \in{ }_{A, G} \mathcal{M}$. 
Final Step. Let $\eta: K \rightarrow L$ be an epimorphism and $\xi: B \otimes_{A} M \rightarrow L$ any morphism in ${ }_{B} \mathcal{M}$. We have to find a morphism $\zeta: B \otimes_{A} M \rightarrow K$ in ${ }_{B} \mathcal{M}$ such that $\xi=\eta \circ \zeta$. Consider the set $\Omega$ of all pairs $(N, \zeta)$ where $N \in \mathcal{G}$ and $\zeta: T_{N} \rightarrow K$ is a morphism in ${ }_{B} \mathcal{M}$ such that $\eta \circ \zeta=\left.\xi\right|_{T_{N}}$. Note that $(0,0) \in \Omega$. For two pairs in $\Omega$ set $(N, \zeta) \leq\left(N^{\prime}, \zeta^{\prime}\right)$ if and only if $N \subset N^{\prime}$ and $\zeta=\left.\zeta^{\prime}\right|_{T_{N}}$. By Zorn's lemma $\Omega$ has a maximal element. Let now $(N, \zeta)$ be such a maximal element. Suppose $N \neq M$. Then there exists $F \in \mathcal{F}$ such that $F \not \subset N$. Put $N^{\prime}=N+F$. By Step $2 T_{N^{\prime}}=V \oplus T_{N}$ for some projective $B$-submodule $V$. The restriction of $\xi$ to $V$ factors through $K$ in ${ }_{B} \mathcal{M}$, and this shows that $\left(N^{\prime}, \zeta^{\prime}\right) \in \Omega$ for a suitable extension $\zeta^{\prime}$ of $\zeta$. We have obtained a contradiction with the maximality of $(N, \zeta)$. Thus $N=M$, whence the required $\zeta$.

Corollary 1.3. Let $M \in{ }_{A, G} \mathcal{M}$ be locally A-finite. If $P$ is a prime ideal of $A$ containing no nonzero $G$-stable ideals, then the localization $M_{P}$ of $M$ at $P$ is a free module over the local ring $A_{P}$. If $A$ is $G$-simple, then $M$ is a projective A-module.

Proof. Apply Theorem 1.2 taking $B=A_{P}$ in one case and $B=A$ in the other. By a well known theorem of Kaplansky all projective modules over a local ring are free [2, Ch. II, §3, Exercise 3].

Corollary 1.4. Suppose $B$ is a $G$-simple commutative algebra, and $A \subset B$ a $G$ stable subalgebra. If $B$ is locally $A$-finite as an object of ${ }_{A, G} \mathcal{M}$, then $B$ is flat over A.

Proof. If $I$ is any nonzero $G$-stable ideal of $A$, then $I B$ is a nonzero $G$-stable ideal of $B$, whence $I B=B$. Suppose that $Q$ is a prime ideal of $B$ and $P=Q \cap A$. Then $P$ can contain no nonzero $G$-stable ideals of $A$, and so $B_{P}$ is a free $A_{P}$-module by Corollary 1.3. Then $B_{Q}$ is flat over $A_{P}$. The flatness of $B$ follows now from [2, Ch. II, §3, Prop. 15].

Examples. 1) Every cocommutative Hopf algebra $H$ determines a formal group scheme $G=\mathfrak{S p}^{*} H$ which can be regarded as a group $k$-functor such that $G(R)$ is the group of all grouplike elements of the Hopf algebra $H \otimes R$ over $R$. Thus

$$
G(R)=\left\{g \in H \otimes R \mid \Delta_{R}(g)=g \otimes g \text { and } \varepsilon_{R}(g)=1\right\} \quad \text { for } R \in C o m m_{k}
$$

where $\Delta_{R}$ and $\varepsilon_{R}$ are the comultiplication and the counit of $H \otimes R$. We follow [40] in considering a formal scheme as a functor defined on the whole $\mathrm{Comm}_{k}$ rather than on the full subcategory consisting of finite dimensional commutative algebras. In this case there is a bijective correspondence between the $G$-module and $H$-module structures. To let $G$ operate on $A$ by automorphisms is the same as to make $A$ into an $H$-module algebra, and one has ${ }_{A, G} \mathcal{M} \approx_{A \# H} \mathcal{M}$. A special case of Theorem 1.2 thus yields

Corollary 1.5. Suppose that $H$ is a cocommutative Hopf algebra, $A$ a commutative $H$-module algebra, and $B$ a commutative $A$-algebra such that $I B=B$ for every $H$ stable ideal $I \neq 0$ of $A$. If $M \in{ }_{A \# H} \mathcal{M}$ is locally $A$-finite, then $B \otimes_{A} M$ is a projective $B$-module.

In particular, one may take $H$ to be a group algebra. In another case where $H$ is the universal enveloping algebra of a Lie algebra one obtains the projectivity result stated in [37, Th. 1.6]. 
2) Every commutative Hopf algebra $H$ determines an affine group scheme $G=$ $\mathfrak{S p} H$ which can be regarded as a group $k$-functor such that $G(R)$ is the set of all algebra homomorphisms $H \rightarrow R$. The multiplication in $G(R)$ comes from the convolution multiplication in $\operatorname{Hom}(H, R)$. The $G$-modules here are precisely right $H$-comodules [5. Ch. II, $\left.\S 2, \mathrm{n}^{\circ} 2.1\right]$. Giving an action of $G$ on $A$ by automorphisms makes $A$ into an $H$-comodule algebra and vice versa. There is a category equivalence ${ }_{A, G} \mathcal{M} \approx \mathcal{M}_{A}^{H}$.

Corollary 1.6. Suppose that $H$ is a commutative Hopf algebra, A a commutative $H$-comodule algebra, and $B$ a commutative $A$-algebra such that $I B=B$ for every $H$-costable ideal $I \neq 0$ of $A$. Then $B \otimes_{A} M$ is a projective $B$-module for any $M \in \mathcal{M}_{A}^{H}$.

We know that $H$ is an $H$-simple $H$-comodule algebra, that is, a $G$-simple algebra in this case. A subalgebra of $H$ is $G$-stable if and only if it is $H$-costable. Corollary 1.4 therefore includes the following result of Masuoka and Wigner [22, Th. 3.4]: $a$ commutative Hopf algebra is a flat module over every right coideal subalgebra. This statement translates into geometric language as follows: if an affine group scheme $G$ operates on an affine scheme $X$, then every $G$-equivariant morphism $f: G \rightarrow X$ decomposes as $G \rightarrow Y \rightarrow X$ where the first morphism is flat and the second one is a closed immersion. Indeed, if $X=\mathfrak{S p} A$, then $f$ corresponds to a homomorphism of $H$-comodule algebras $\varphi: A \rightarrow H$. The image of $\varphi$ is a right coideal subalgebra of $H$ over which $H$ is flat. We may therefore take $Y=\mathfrak{S p} \varphi(A)$. In a special case where $f$ is a homomorphism of affine group schemes, $f$ induces a faithfully flat homomorphism of $G$ onto a closed group subscheme of $X$ [5, Ch. III, §3, Cor. 7.3]. A purely algebraic formulation of the latter fact says that a commutative Hopf algebra is a faithfully flat module over every Hopf subalgebra [39, Th. 3.1]. The morphism $G \rightarrow Y$ in the decomposition of $f$ above is also always faithfully flat under the assumption that $X$ is a finite scheme. Indeed, as was announced by Masuoka 20, Th. 3.5] (and follows also from Theorem 6.1 later in the paper), a commutative Hopf algebra is a free module over every finite dimensional right coideal subalgebra. In case of Hopf subalgebras the last statement was proved by Radford [32].

\section{SOME RING-THEORETIC FACTS}

Recall that a ring is semilocal if its factor ring by the Jacobson radical is semisimple Artinian. Following [33. Def. 1.3.30] we say that a ring $R$ is weakly $n$-finite if for every pair of $n \times n$-matrices $X, Y \in \operatorname{Mat}_{n}(R)$ the equality $X Y=1$ in the matrix ring implies the equality $Y X=1$. A ring $R$ is weakly finite (or stably finite according to different sources, e.g., [24]) if $R$ is weakly $n$-finite for all integers $n>0$.

Lemma 2.1. Let $A$ be a ring, and $M$ a finitely generated right A-module. The ring $R=\operatorname{End}_{A} M$ is weakly finite in each of the following three cases:

(a) every finite subset of $A$ is contained in a right Noetherian subring,

(b) $A$ is commutative,

(c) $A$ is weakly finite and $M$ is projective.

Proof. Let $\xi, \eta \in R$ be such that $\xi \circ \eta=$ id. Pick any generators $e_{1}, \ldots, e_{m}$ for $M$ over $A$. Under hypothesis (a) there exists a Noetherian subring $B \subset A$ such that $\xi\left(e_{1}\right), \ldots, \xi\left(e_{m}\right)$ and $\eta\left(e_{1}\right), \ldots, \eta\left(e_{m}\right)$ lie in the $B$-submodule $N \subset M$ generated by $e_{1}, \ldots, e_{m}$. Then $N$ is stable under both $\xi$ and $\eta$. In particular, $\xi$ induces a 
surjective endomorphism of $N$. Since $N$ is a Noetherian $B$-module, $\xi$ has to be bijective on $N$ [11, Th. 6.4.1], and it follows that $\eta \circ \xi$ is identity on $N$. Since $M=N A$, we conclude that $\eta \circ \xi=$ id on the whole $M$. Note that hypothesis (b) is a special case of (a). Indeed, finitely generated commutative rings are all Noetherian. Under hypothesis (c) $M$ is a direct summand of a free right $A$-module $A^{t}$ for some integer $t \geq 0$. Letting both $\xi$ and $\eta$ act as identity on a complementary summand, we extend $\xi$ and $\eta$ to endomorphisms of $A^{t}$ so that $\xi \circ \eta=$ id on $A^{t}$. Since the $\operatorname{ring} \operatorname{End}_{A} A^{t} \cong \operatorname{Mat}_{t}(A)$ is weakly 1-finite, the equality $\eta \circ \xi=$ id holds again.

We have checked that $R$ is weakly 1 -finite in each of cases (a), (b), (c). It remains to observe that $\operatorname{Mat}_{n}(R) \cong \operatorname{End}_{A} M^{n}$ and $M^{n}$ is a finitely generated right $A$-module which is also projective in case (c).

Proposition 2.2. A ring $R$ is weakly finite in each of the cases listed below:

(a) $R$ is left or right Noetherian,

(b) $R$ is semilocal,

(c) $R^{\text {op }}$ is weakly finite,

(d) $R$ is a finitely generated left or right module over a commutative subring $S$,

(e) $R$ is a finitely generated projective left or right module over a weakly finite subring $S$,

(f) $R=A \otimes B$ where $A, B$ are algebras over the ground field $k$ such that $A$ is a finitely generated module over its center $Z$ and $B$ is weakly finite.

Part (f) is a special case of Montgomery's result [24, Th. 1] where the assumption on $A$ is weakened to $A$ being any polynomial identity algebra. We nevertheless provide a proof of (f) as it uses a different argument.

Proof. Part (a) is covered by [24, Cor. 1] or [33, Th. 3.2.37].

(b) Let $J$ be the Jacobson radical of $R$. The ring $R / J$ is Artinian, hence weakly finite. Then $R$ is weakly finite too [24, Lemma 2].

(c) Use an isomorphism $\operatorname{Mat}_{n}\left(R^{\mathrm{op}}\right) \cong \operatorname{Mat}_{n}(R)^{\mathrm{op}}$.

(d), (e) There are embeddings $R \subset$ End $R_{S}$ and $R \subset\left(\text { End }_{S} R\right)^{\mathrm{op}}$. Now we can apply Lemma 2.1 and (c).

(f) Since $\operatorname{Mat}_{n}(R) \cong A \otimes \operatorname{Mat}_{n}(B)$ and the ring $\operatorname{Mat}_{n}(B)$ is weakly finite in view of (e), it suffices to prove that $R$ is weakly 1 -finite. Let $e_{1}, \ldots, e_{m}$ generate $A$ as a $Z$-module. There exists a finitely generated subalgebra $Z^{\prime} \subset Z$ such that $e_{i} e_{j} \in \sum_{l=1}^{m} Z^{\prime} e_{l}$ for all $1 \leq i, j \leq m$. Then $\sum_{l=1}^{m} K e_{l}$ is a subalgebra of $A$ whenever $K$ is a subalgebra satisfying $Z^{\prime} \subset K \subset Z$. This shows that every finite subset of $A$ is contained in a subalgebra $A^{\prime} \subset A$ which is finitely generated as a module over some finitely generated central subalgebra $K \subset A^{\prime}$. Given $x, y \in R$, there exists $A^{\prime}$ as above such that $x, y \in A^{\prime} \otimes B$. Suppose that $x y=1$. If $I$ is any ideal of $K$ such that $\operatorname{dim} K / I<\infty$, then $\operatorname{dim} A^{\prime} / I A^{\prime}<\infty$ as well. The ring $A^{\prime} / I A^{\prime} \otimes B$ is weakly finite by (e) as it is a finitely generated free module over its subring $1 \otimes B$. Considering the images of $x, y$ in $A^{\prime} / I A^{\prime} \otimes B$, we deduce that $y x-1 \in I A^{\prime} \otimes B$. Hence $y x-1$ lies in the intersection $\bigcap I A^{\prime} \otimes B$ over all ideals $I$ of $K$ satisfying $\operatorname{dim} K / I<\infty$. It remains to check that $\bigcap I A^{\prime}=0$. If $P \in \operatorname{Max} K$, then $\operatorname{dim} K / P<\infty$ by Hilbert's Nullstellensatz. As $K$ is Noetherian, we have also $\operatorname{dim} K / P^{i}<\infty$ for all $i>0$. For an element $a \in A^{\prime}$ denote $A n n(a)=\{z \in K \mid z a=0\}$. By [2, Ch. III, §3, Prop. 5] the inclusion $a \in \bigcap_{i>0} P^{i} A^{\prime}$ implies that $\operatorname{Ann}(a) \not \subset P$, and if this is valid for all $P \in \operatorname{Max} K$ then $\operatorname{Ann}(a)=K$, which is only possible for $a=0$. 
Let $M$ be a finitely generated right $R$-module. For every system of its generators $e_{1}, \ldots, e_{n}$ denote by $\mathcal{I}_{e_{1}, \ldots, e_{n}}$ the ideal of $R$ generated by all elements of $R$ which occur as a coefficient in a zero linear combination $e_{1} x_{1}+\cdots+e_{n} x_{n}=0$ with $x_{1}, \ldots, x_{n} \in R$. If $R$ is commutative, then clearly $\mathcal{I}_{e_{1}, \ldots, e_{n}}=\operatorname{Fitt}_{n-1}(M)$.

Lemma 2.3. Let $e_{1}, \ldots, e_{n}$ and $e_{1}^{\prime}, \ldots, e_{n}^{\prime}$ be two systems of generators for $M$ having the same number of elements. Suppose that $I$ is an ideal of $R$ such that $\mathcal{I}_{e_{1}, \ldots, e_{n}} \subset I$. If the ring $R / I$ is weakly $n$-finite, then $\mathcal{I}_{e_{1}^{\prime}, \ldots, e_{n}^{\prime}} \subset I$.

Proof. There are expressions $e_{j}^{\prime}=\sum_{i=1}^{n} e_{i} a_{i j}$ and $e_{j}=\sum_{i=1}^{n} e_{i}^{\prime} b_{i j}$ for some $a_{i j}, b_{i j} \in$ $R$ with $1 \leq i, j \leq n$. Then $e_{l}=\sum_{j=1}^{n} \sum_{i=1}^{n} e_{i} a_{i j} b_{j l}$, i.e.

$$
\sum_{i=1}^{n} e_{i}\left(\sum_{j=1}^{n} a_{i j} b_{j l}-\delta_{i l}\right)=0
$$

for each $l=1, \ldots, n$. This shows that $\sum_{j=1}^{n} a_{i j} b_{j l}-\delta_{i l} \in I$ for all $i, l$. Denote by $X, Y \in \operatorname{Mat}_{n}(R / I)$ the $n \times n$ matrices whose entries are the cosets, respectively, of elements $a_{i j}$ and $b_{i j}$ modulo $I$. Previous inclusions can be rewritten as a single matrix equality telling us that $X Y$ is the identity matrix in $\operatorname{Mat}_{n}(R / I)$. By the hypotheses of the lemma so then is the product $Y X$ as well, i.e.

$$
\sum_{j=1}^{n} b_{i j} a_{j l}-\delta_{i l} \in I \quad \text { for all } 1 \leq i, l \leq n .
$$

Suppose now that $\sum_{l=1}^{n} e_{l}^{\prime} y_{l}=0$ for some $y_{1}, \ldots, y_{n} \in R$. Expressing each $e_{l}^{\prime}$ as a linear combination of $e_{1}, \ldots, e_{n}$, we get $\sum_{j=1}^{n} e_{j}\left(\sum_{l=1}^{n} a_{j l} y_{l}\right)=0$ so that $\sum_{l=1}^{n} a_{j l} y_{l} \in I$ for all $j=1, \ldots, n$. Since $I$ is a two-sided ideal, preceding inclusions together with $(*)$ yield

$$
y_{i}=\sum_{j=1}^{n} b_{i j} \sum_{l=1}^{n} a_{j l} y_{l}-\sum_{l=1}^{n}\left(\sum_{j=1}^{n} b_{i j} a_{j l}-\delta_{i l}\right) y_{l} \in I
$$

for all $i=1, \ldots, n$. The inclusion asserted by the lemma now follows from the definition of $\mathcal{I}_{e_{1}^{\prime}, \ldots, e_{n}^{\prime}}$.

Lemma 2.4. Let $R$ be a semilocal ring and $\mathcal{V}$ the class of all finitely generated projective right $R$-modules $V$ such that a direct sum of finitely many copies of $V$ is a free $R$-module. In order that $V \in \mathcal{V}$ be a free $R$-module it is necessary and sufficient that $V / V Q$ be a free $R / Q$-module for at least one $Q \in$ Max $R$. There exists $U \in \mathcal{V}$ such that all modules in $\mathcal{V}$ are direct sums of copies of $U$.

Proof. Denote by $J$ the Jacobson radical of $R$. Suppose that $V$ is a finitely generated projective right $R$-module. We have $V / V J \cong \prod_{Q \in \operatorname{Max} R} V / V Q$, and each $V / V Q$ is a direct sum of finitely many copies of the single simple $R / Q$-module. Put

$$
r_{Q}(V)=\frac{\operatorname{lng} V / V Q}{\operatorname{lng} R / Q} \quad \text { for } Q \in \operatorname{Max} R
$$

where $\operatorname{lng}$ is short for length. In order that $V$ be a free $R$-module of rank $n$ it is necessary and sufficient that $V / V J$ be a free $R / J$-module of rank $n$ 2, Ch. II, $\S 3$, Prop. 5]. The latter condition means precisely that each $V / V Q$ is a free $R / Q$ module of rank $n$ or, equivalently, $r_{Q}(V)=n$ for each $Q$. If $V^{t}$ is a free $R$-module of rank $m$ for some integers $t>0$ and $m \geq 0$, then $t \operatorname{lng} V / V Q=m \operatorname{lng} R / Q$, and 
so $r_{Q}(V)=m / t$ for all $Q \in \operatorname{Max} R$. This shows that the numbers $r_{Q}(V)$ for $V \in \mathcal{V}$ do not depend on $Q$. Let $r(V)$ denote the common value of these numbers. We see that $V \in \mathcal{V}$ is free if and only if $r(V) \in \mathbb{Z}$. This proves the first assertion.

We claim that, whenever $r(V) \geq r(W)$ for some $V, W \in \mathcal{V}$, there always exists an epimorphism $V \rightarrow W$. First of all, there exists an epimorphism $V / V Q \rightarrow W / W Q$ for each $Q \in \operatorname{Max} R$, hence an epimorphism $V / V J \rightarrow W / W J$. The latter can be lifted to a homomorphism $\xi: V \rightarrow W$ since $V$ is projective. By Nakayama's lemma $\xi$ is surjective.

We can find an integer $d>0$ such that $r(V) \in \frac{1}{d} \mathbb{Z}$ for all $V \in \mathcal{V}$. For instance, we may take $d=\operatorname{lng} R / Q$ for any chosen $Q \in \operatorname{Max} R$. It follows that there exists $0 \neq U \in \mathcal{V}$ such that $r(U) \leq r(V)$ for all $0 \neq V \in \mathcal{V}$. Given $V$, let $n \geq 0$ be the largest integer such that $r\left(U^{n}\right)=r(U) n \leq r(V)$. As we have seen, $U^{n}$ is a homomorphic image of $V$, so that $V \cong U^{n} \oplus T$ for some projective $R$-module $T$. Now $r_{Q}(T)=r(V)-r\left(U^{n}\right)$ for all $Q \in \operatorname{Max} R$, and so $r_{Q}\left(T^{d}\right)$ is an integer not depending on $Q$. This shows that $T^{d}$ is a free $R$-module, whence $T \in \mathcal{V}$. By the choice of $n$ we must have $r(T)<r(U)$, but then $T=0$ and $V \cong U^{n}$.

Lemma 2.5. Let $R$ be a semilocal ring. A right $R$-module $M$ is necessarily free as long as $M$ is not finitely generated and there exists a family $\mathcal{F}$ of its submodules satisfying conditions (a) and (b) below:

(a) $0 \in \mathcal{F}$ and the union of every chain in $\mathcal{F}$ is again in $\mathcal{F}$,

(b) each $N \in \mathcal{F}, N \neq M$, is properly contained in some $N^{\prime} \in \mathcal{F}$ with $N^{\prime} / N \in \mathcal{V}$.

Proof. Let $U$ be as in Lemma 2.4. For each subset $X \subset \operatorname{Hom}_{R}(U, M)$ denote by $U^{(X)}$ the direct sum of the family of copies of $U$ indexed by $X$. For $\xi \in X$ let $\iota_{\xi}: U \rightarrow U^{(X)}$ be the canonical embedding of a summand. There exists a unique homomorphism $\theta_{X}: U^{(X)} \rightarrow M$ such that $\theta_{X} \circ \iota_{\xi}=\xi$ for each $\xi$.

Denote by $\Omega$ the set of all pairs $(N, X)$ where $N \in \mathcal{F}$ and $X \subset \operatorname{Hom}_{R}(U, M)$ is a subset such that $\theta_{X}$ is an isomorphism onto $N$. Note that $(0, \varnothing) \in \Omega$. Define a partial order on $\Omega$ by setting $(N, X) \leq\left(N^{\prime}, X^{\prime}\right)$ for two pairs in $\Omega$ if and only if $N \subset N^{\prime}$ and $X \subset X^{\prime}$. If $\left\{\left(N_{\alpha}, X_{\alpha}\right)\right\}$ is a chain in $\Omega$ indexed by elements $\alpha$ of some set, then ( $\left.\bigcup N_{\alpha}, \bigcup X_{\alpha}\right)$ is in $\Omega$ according to (a). By Zorn's lemma $\Omega$ has a maximal element. Now let $(N, X)$ be such a maximal element. Suppose that $N \neq M$, and let $N^{\prime}$ be as in (b). We then have an isomorphism of $R$-modules $N^{\prime} \cong N \oplus V$ where $V=N^{\prime} / N \in \mathcal{V}$. By Lemma $2.4 V \cong U^{n}$ for some integer $n \geq 0$. Let $\eta_{1}, \ldots, \eta_{n}$ be the isomorphisms of $U$ onto direct summands in the decomposition of $V$. Denote $X^{\prime}=X \cup\left\{\eta_{1}, \ldots, \eta_{n}\right\}$. Then $\left(N^{\prime}, X^{\prime}\right) \in \Omega$ and $(N, X)<\left(N^{\prime}, X^{\prime}\right)$. We have arrived at a contradiction with the maximality of $(N, X)$.

It follows that any maximal element of $\Omega$ is necessarily $(M, X)$ for some $X$. Thus $M \cong U^{(X)}$. Since $M$ is not finitely generated, $X$ must be infinite. Then $X$ has the same cardinality as the set $X \times\{1, \ldots, t\}$ for any integer $t>0$. However, $U^{t}$ is a free $R$-module for a suitable $t$. It follows that $U^{(X)} \cong R^{(X)}$.

\section{Projectivity Result for COModule Algebras}

Let $A$ be an $H$-comodule algebra. In the main result of this section we encounter the following technical condition on $A$ :

(C) the ring $A / Q \otimes H$ is weakly finite for each $Q \in \operatorname{Max} A$. 
Using Proposition 2.2 one can verify the validity of this condition in many cases. For instance, (C) always holds whenever $H$ is weakly finite and $A$ is finitely generated as a module over its center. If $\operatorname{dim} H<\infty$, condition $(\mathrm{C})$ is fulfilled provided that $A$ is semilocal. The same is true if $H$ is only residually finite dimensional in the sense that its ideals of finite codimension have zero intersection.

Lemma 3.1. If $e_{1}, \ldots, e_{n}$ generate $M \in \mathcal{M}_{A}^{H}$ as an $A$-module, then $\rho\left(e_{1}\right), \ldots, \rho\left(e_{n}\right)$ generate $M \otimes H$ as an $A \otimes H$-module.

Proof. If $m \in M$, then

$$
m \otimes 1=\sum_{(m)} m_{(0)} \otimes m_{(1)} s\left(m_{(2)}\right)=\sum_{(m)} \rho\left(m_{(0)}\right) \cdot\left(1 \otimes s\left(m_{(1)}\right)\right),
$$

which shows that $M \otimes H=\rho(M) \cdot(A \otimes H)$. Since $\rho(m a)=\rho(m) \rho(a)$ for $m \in M$ and $a \in A$, we see that $\rho(M)$ is generated by $\rho\left(e_{1}\right), \ldots, \rho\left(e_{n}\right)$ as a $\rho(A)$-module, and the lemma is proved.

Lemma 3.2. Let $M \in \mathcal{M}_{A}^{H}$ be generated by $e_{1}, \ldots, e_{n}$ as an A-module. Suppose that $I$ is an ideal of $A$ such that $\mathcal{I}_{e_{1}, \ldots, e_{n}} \subset I$ and the ring $A / I \otimes H$ is weakly $n$-finite. Then there exists an $H$-costable ideal $K$ of $A$ such that $\mathcal{I}_{e_{1}, \ldots, e_{n}} \subset K \subset I$.

Proof. Put $J=\mathcal{I}_{e_{1}, \ldots, e_{n}}$ (as defined in Lemma 2.3) and $K=\rho^{-1}(I \otimes H)$. Since $\rho: A \rightarrow A \otimes H$ is an algebra homomorphism, $K$ is an ideal of $A$. One has $K \subset I$ because (id $\otimes \varepsilon) \circ \rho=$ id and $\rho(K) \subset K \otimes H$ because

$$
(\rho \otimes \mathrm{id}) \circ \rho(K)=(\mathrm{id} \otimes \Delta) \circ \rho(K) \subset(\mathrm{id} \otimes \Delta)(I \otimes H) \subset I \otimes H \otimes H .
$$

The $A \otimes H$-module $M \otimes H$ is clearly generated by $e_{1} \otimes 1, \ldots, e_{n} \otimes 1$. It is straightforward to see that $\mathcal{I}_{e_{1} \otimes 1, \ldots, e_{n} \otimes 1}=J \otimes H$. By Lemma $3.1 \rho\left(e_{1}\right), \ldots, \rho\left(e_{n}\right)$ is another system of generators for the $A \otimes H$-module $M \otimes H$. Applying Lemma 2.3 with $R=A \otimes H$ and the ideal $I \otimes H$, we get $\mathcal{I}_{\rho\left(e_{1}\right), \ldots, \rho\left(e_{n}\right)} \subset I \otimes H$. Now if $\sum_{i=1}^{n} e_{i} x_{i}=0$ for some $x_{1}, \ldots, x_{n} \in A$, then $\sum_{i=1}^{n} \rho\left(e_{i}\right) \rho\left(x_{i}\right)=0$ in $M \otimes H$, which shows that $\rho\left(x_{i}\right) \in \mathcal{I}_{\rho\left(e_{1}\right), \ldots, \rho\left(e_{n}\right)}$, and therefore $x_{i} \in K$ for all $i=1, \ldots, n$. It follows that $J \subset K$ by the definition of $J$.

Lemma 3.3. Let $M \in \mathcal{M}_{A}^{H}$ be generated by $e_{1}, \ldots, e_{n}$ as an A-module. Suppose that $I$ is an ideal of $A$ such that the ring $A / I \otimes H$ is weakly $n$-finite and $I$ contains no nonzero $H$-costable ideals of $A$. If the cosets of $e_{1}, \ldots, e_{n}$ give a basis for the $A / I$-module $M / M I$, then $e_{1}, \ldots, e_{n}$ are a basis for the A-module $M$.

Proof. Any relation $\sum_{i=1}^{n} e_{i} x_{i}=0$ implies $x_{1}, \ldots, x_{n} \in I$ by the freeness of $M / M I$. It follows that $\mathcal{I}_{e_{1}, \ldots, e_{n}} \subset I$. Applying Lemma 3.2 , we have $K=0$ by the assumptions about $I$. Hence $\mathcal{I}_{e_{1}, \ldots, e_{n}}=0$, which means that $e_{1}, \ldots, e_{n}$ are linearly independent over $A$.

In the next lemma we denote by $\operatorname{lng} V$ the length of $V \in \mathcal{M}_{A}$ and for each $Q \in \operatorname{Max} A$ put

$$
r_{Q}(M)=\frac{\operatorname{lng} M / M Q}{\operatorname{lng} A / Q} .
$$

Lemma 3.4. Let $A$ be semilocal. Suppose that $M \in \mathcal{M}_{A}^{H}$ is $A$-finite and there exists $P \in \operatorname{Max} A$ such that $P$ contains no nonzero $H$-costable ideals of $A$, the ring 
$A / P \otimes H$ is weakly finite and $r_{P}(M) \geq r_{Q}(M)$ for all $Q \in$ Max $A$. Then:

(i) a suitable direct sum of finitely many copies of $M$ is a free A-module,

(ii) if $r_{P}(M) \in \mathbb{Z}$, then $M$ is a free $A$-module.

By the hypotheses

$$
\operatorname{lng}(A / Q)^{n}=n \operatorname{lng} A / Q \geq \operatorname{lng} M / M Q
$$

for each $Q \in \operatorname{Max} A$. Since $A / Q$ is a simple Artinian ring, we deduce that the $A / Q$-module $M / M Q$ is an epimorphic image of $(A / Q)^{n}$, and so $M / M Q$ can be generated by $n$ elements. Moreover, $M / M P \cong(A / P)^{n}$ is a free $A / P$-module. Denote by $J$ the Jacobson radical of $A$. Since $M / M J \cong \prod_{Q \in \operatorname{Max} A} M / M Q$, we can find elements $e_{1}, \ldots, e_{n} \in M$ whose images generate the $A / Q$-module $M / M Q$ for each $Q$ and give a basis for the $A / P$-module $M / M P$. Then $e_{1}, \ldots, e_{n}$ generate the $A$-module $M$ by Nakayama's lemma. Taking $I=P$, we meet the hypotheses of Lemma 3.3 which yields (ii).

In general let $N \in \mathcal{M}_{A}^{H}$ be the direct sum of $t$ copies of $M$ where $t>0$ is an integer such that $r_{P}(M) t \in \mathbb{Z}$. We can apply (ii) to $N$.

Theorem 3.5. Suppose that $A$ is a semilocal H-simple H-comodule algebra satisfying $(\mathrm{C})$. Then all objects of $\mathcal{M}_{A}^{H}$ are projective A-modules. Moreover, $M \in \mathcal{M}_{A}^{H}$ is a free $A$-module if and only if $M / M Q$ is a free $A / Q$-module for at least one $Q \in \operatorname{Max} A$.

Proof. If $M$ is $A$-finite, then $M$ fulfills the hypotheses of Lemma 3.4 with $P$ taken to be any maximal ideal of $A$ for which $r_{P}(M)$ attains the maximum value. Denote by $\mathcal{V}$ the class of all right $A$-modules $V$ such that a direct sum of finitely many copies of $V$ is a free $A$-module. Lemma 3.4 thus shows that all $A$-finite objects of $\mathcal{M}_{A}^{H}$ belong to $\mathcal{V}$. In particular, they are direct summands of free $A$-modules, hence projective. We also see that the family $\mathcal{F}$ of all $\mathcal{M}_{A}^{H}$-subobjects of an arbitrary $M \in \mathcal{M}_{A}^{H}$ satisfies conditions (a) and (b) of Lemma 2.5 where we take $R=A$. Indeed, if $N \in \mathcal{F}$ and $N \neq M$, then there exists an $A$-finite $L \in \mathcal{F}$ such that $L \not \subset N$. Then $N^{\prime}=N+L$ belongs to $\mathcal{F}$ and properly contains $N$, while $N^{\prime} / N \in \mathcal{M}_{A}^{H}$ is $A$-finite. Lemmas 2.4 and 2.5 complete the proof. In particular, if $M$ is not $A$-finite, then $M$ is a free $A$-module.

Corollary 3.6. Under the hypotheses of Theorem 3.5 suppose also that $A / Q$ is a skew field for some $Q \in \operatorname{Max} A$. Then:

(i) all objects of $\mathcal{M}_{A}^{H}$ are free $A$-modules,

(ii) $A$ is a simple object of $\mathcal{M}_{A}^{H}$,

(iii) $A^{H}$ is a skew field.

Proof. Part (i) follows from the fact that $M / M Q$ is a free $A / Q$-module for any $M \in \mathcal{M}_{A}^{H}$. If $N \subset M$ is an $\mathcal{M}_{A}^{H}$-subobject, then $M \cong N \oplus M / N$ in $\mathcal{M}_{A}$ where both $N$ and $M / N$ are free $A$-modules. If $0 \neq N \neq M$, then the $A$-module $M$ has a basis consisting of at least two elements. This proves (ii) since every semilocal ring has invariant base number. The left multiplication by an element $a \in A$ commutes with the coaction of $H$ if and only if $a \in A^{H}$. This shows that $A^{H}$ is isomorphic to the endomorphism ring of $A$ as an object of $\mathcal{M}_{A}^{H}$. Now (iii) is a consequence of (ii) and Schur's Lemma (cf. [1, Lemma 2.1]). 
Proposition 3.7. Suppose that $A$ is a semilocal H-comodule algebra satisfying $(\mathrm{C})$ and having a minimal nonzero $H$-costable ideal $M$ which is finitely generated in $\mathcal{M}_{A}$. If at least one maximal ideal of $A$ contains no nonzero $H$-costable ideals of $A$, then $A$ is $H$-simple.

Proof. We may regard $M$ as an $A$-finite object of $\mathcal{M}_{A}^{H}$. If $I$ is any nonzero $H$ costable ideal of $A$, then $M I \neq 0$ because there exists a maximal ideal of $A$ containing neither $M$ nor $I$. Then $M I=M$ by the minimality of $M$. Denote by $\Omega \subset \operatorname{Max} A$ the subset of those maximal ideals of $A$ which contain a nonzero $H$ costable ideal of $A$. We see that $M Q=M$ for each $Q \in \Omega$. Then $r_{Q}(M)=0$ whenever $Q \in \Omega$. By the hypotheses $\Omega \neq \operatorname{Max} A$. It follows that the maximum of the numbers $r_{Q}(M), Q \in \operatorname{Max} A$, is attained at some maximal ideal $P \notin \Omega$. We can now apply Lemma 3.4 which shows that $M^{t}$ is a nonzero free $A$-module for some integer $t>0$. Then $(M / M Q)^{t}$ is a nonzero free $A / Q$-module, so that $M Q \neq M$, for each $Q \in \operatorname{Max} A$. It follows that $\Omega=\varnothing$, and this gives the desired conclusion.

Corollary 3.8. Let $R$ be a finite dimensional simple algebra, and $A$ a finite dimensional $H$-simple $H$-comodule algebra. Suppose that $A / P$ is a central simple algebra for some $P \in$ Max $A$ and $H$ is weakly finite. Then $R \otimes A$ is an $H$-simple H-comodule algebra with respect to the comodule structure id $\otimes \rho: R \otimes A \rightarrow R \otimes A \otimes H$.

Proof. The algebra $R \otimes A / P$ is simple by [33, Th. 1.7.27]. Hence $P^{\prime}=R \otimes P$ is a maximal ideal of $B=R \otimes A$. Take any basis $e_{1}, \ldots, e_{m}$ for $R$ and define $\pi_{1}, \ldots, \pi_{m}: B \rightarrow A$ such that $x=\sum_{i=1}^{m} e_{i} \otimes \pi_{i}(x)$ for each $x \in B$. Clearly each $\pi_{i}$ is a morphism in $\mathcal{M}^{H}$. Suppose that $V \subset P^{\prime}$ is an $H$-subcomodule of $B$. Then $\pi_{i}(V) \subset P$ is an $H$-subcomodule of $A$. The ideal of $A$ generated by $\pi_{i}(V)$ is $H$ costable and is contained in $P$. It follows that $\pi_{i}(V)=0$ for each $i=1, \ldots, m$ by the hypotheses. Thus $P^{\prime}$ contains no nonzero $H$-costable ideals of $B$. Since $\operatorname{dim} B<\infty$, we can apply Proposition 3.7 to the $H$-comodule algebra $B$.

\section{When are COMOdUle Algebras QUASI-Frobenius?}

Assume throughout this section that $A$ is an $H$-comodule algebra. For finite dimensional algebras we can readily employ duality to derive additional information. The next lemma generalizes [19, Lemma 2.10].

Lemma 4.1. Let $M \in{ }_{A} \mathcal{M}^{H}$. If either $\operatorname{dim} M<\infty$ or $\operatorname{dim} H<\infty$, then the dual vector space $M^{*}$ is an object of $\mathcal{M}_{A}^{H}$ in a canonical way.

Proof. Let $\nu: M^{*} \rightarrow \operatorname{Hom}(M, H)$ be the map that assigns to $\xi \in M^{*}$ the composite $M \stackrel{\rho}{\longrightarrow} M \otimes H \stackrel{\xi \otimes \mathrm{id}}{\longrightarrow} H$. By the hypotheses $\operatorname{Hom}(M, H) \cong H \otimes M^{*}$, and $\nu$ is a left $H$-comodule structure on $M^{*}$. Composing $\nu$ with the map $H \otimes M^{*} \rightarrow M^{*} \otimes H$ given by $h \otimes \xi \mapsto \xi \otimes s(h)$, we obtain a right $H$-comodule structure on $M^{*}$. If $U \in \mathcal{M}^{H}$, then the canonical isomorphism $\operatorname{Hom}(U \otimes M, k) \cong \operatorname{Hom}\left(U, M^{*}\right)$ induces a bijective correspondence between the morphisms $U \otimes M \rightarrow k$ and $U \rightarrow M^{*}$ in $\mathcal{M}^{H}$ where $U \otimes M$ is given the tensor product of comodule structures and $k$ is the trivial $H$-comodule. 
Clearly $M^{*}$ is also a right $A$-module with respect to the action $(\xi a)(\mathrm{m})=\xi(\mathrm{am})$ where $\xi \in M^{*}, a \in A$ and $m \in M$. Now there is a commutative diagram

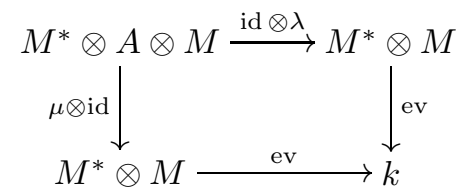

where $\lambda: A \otimes M \rightarrow M$ and $\mu: M^{*} \otimes A \rightarrow M^{*}$ are the $A$-module structures. Both $\lambda$ and the evaluation map ev : $M^{*} \otimes M \rightarrow k$ are morphisms in $\mathcal{M}^{H}$. Hence so too is the composite $\varphi=$ ev $\circ($ id $\otimes \lambda)$. We may regard $\varphi$ as a linear map $U \otimes M \rightarrow k$ with $U=M^{*} \otimes A$. The diagram shows that $\varphi$ corresponds to $\mu: U \rightarrow M^{*}$. It follows that $\mu$ is a morphism in $\mathcal{M}^{H}$, which yields the required compatibility of module and comodule structures on $M^{*}$.

Theorem 4.2. Let $A$ be a finite dimensional $H$-simple $H$-comodule algebra. If $H$ is weakly finite, then:

(i) $A$ is Frobenius,

(ii) all objects of both $\mathcal{M}_{A}^{H}$ and ${ }_{A} \mathcal{M}^{H}$ are projective $A$-modules,

(iii) $M \in \mathcal{M}_{A}^{H}$ (resp. $M \in{ }_{A} \mathcal{M}^{H}$ ) is a free $A$-module if and only if $M / M Q$ (resp. $M / Q M)$ is a free $A / Q$-module for at least one $Q \in \operatorname{Max} A$.

Proof. By Proposition 2.2 A satisfies (C). Now the assertions concerning $\mathcal{M}_{A}^{H}$ are restatements of Theorem 3.5. If $M \in{ }_{A} \mathcal{M}^{H}$ is $A$-finite, then $\operatorname{dim} M<\infty$, and so $M^{*}$ is a projective right $A$-module in view of Lemma 4.1. Moreover, $\left(M^{*}\right)^{l}$ is a free $A$-module for some integer $l \geq 0$. In particular, we may regard $A$ as an object of ${ }_{A} \mathcal{M}^{H}$. Comparing dimensions, we deduce that $\left(A^{*}\right)^{t} \cong A^{t}$ in $\mathcal{M}_{A}$ for some $t>0$. By the Krull-Schmidt theorem $A^{*} \cong A$ in $\mathcal{M}_{A}$, which yields (i). We then have $A^{*} \cong A$ in ${ }_{A} \mathcal{M}$ as well, and so the linear duals of free right $A$-modules are free left $A$-modules. Continuing with an $A$-finite $M \in{ }_{A} \mathcal{M}^{H}$, we conclude that $M^{l}$ is a free $A$-module for $l$ as above. The proof of (ii) and (iii) is now completed by Lemmas 2.4 and 2.5 .

Given $U \in \mathcal{M}_{H}$ and $V \in \mathcal{M}_{A}$, we regard $V \otimes U$ as a right $A \otimes H$-module with respect to the action $(v \otimes u)(a \otimes h)=v a \otimes u h$ where $v \in V, u \in U, a \in A$ and $h \in H$. Then $V \otimes U$ is a right $A$-module via the algebra homomorphism $\rho: A \rightarrow A \otimes H$. Define a right $H$-module structure on the dual of $U$ by the rule $\langle u, \xi h\rangle=\langle u s(h), \xi\rangle$ for $u \in U, \xi \in U^{*}$ and $h \in H$.

Lemma 4.3. For $U \in \mathcal{M}_{H}$ and $V, W \in \mathcal{M}_{A}$ there is a canonical linear injection

$$
\Phi: \operatorname{Hom}_{A}(W, V \otimes U) \rightarrow \operatorname{Hom}_{A}\left(W \otimes U^{*}, V\right)
$$

which is a bijection whenever $\operatorname{dim} U<\infty$. More generally, let $X \subset U$ be any finite dimensional subspace. Then $\Phi$ establishes a bijective correspondence between the A-module homomorphisms $W \rightarrow V \otimes U$ whose image is contained in $V \otimes X$ and the A-module homomorphisms $W \otimes U^{*} \rightarrow V$ which factor through $W \otimes X^{*}$.

Proof. Assigning the composite $W \otimes T^{*} \stackrel{\theta \otimes \mathrm{id}}{\longrightarrow} V \otimes T \otimes T^{*} \stackrel{\mathrm{id} \otimes \mathrm{ev}_{T}}{\longrightarrow} V$ to each linear map $\theta: W \rightarrow V \otimes T$, where $T$ is any vector space and $\operatorname{ev}_{T}: T \otimes T^{*} \rightarrow k$ the 
evaluation map $t \otimes \xi \mapsto\langle t, \xi\rangle$, we obtain a linear map

$$
\Phi_{T}: \operatorname{Hom}(W, V \otimes T) \rightarrow \operatorname{Hom}\left(W \otimes T^{*}, V\right) .
$$

Clearly $\Phi_{T}$ is injective. If $\operatorname{dim} T<\infty$, then $\Phi_{T}$ is bijective.

Note that $\mathrm{ev}_{U}: U \otimes U^{*} \rightarrow k$ is a morphism in $\mathcal{M}_{H}$, and it follows that the map $\Phi_{U}(\theta): W \otimes U^{*} \rightarrow V$ is a morphism in $\mathcal{M}_{A}$ whenever so is $\theta: W \rightarrow V \otimes U$. Since the maps $\Phi_{T}$ are natural in $T$, we see that $\Phi_{U}$ induces a bijection between the linear maps $W \rightarrow V \otimes U$ whose image is contained in $V \otimes X$ and the linear maps $W \otimes U^{*} \rightarrow V$ vanishing on $W \otimes X^{\perp}$ where $X^{\perp}$ denotes the kernel of the restriction map $U^{*} \rightarrow X^{*}$. Suppose that $\eta: W \otimes U^{*} \rightarrow V$ is a morphism in $\mathcal{M}_{A}$ such that $\eta\left(W \otimes X^{\perp}\right)=0$, and let $\theta=\Phi_{U}^{-1}(\eta)$. One computes $\theta$ by the formula

$$
\theta(w)=\sum_{i=1}^{n} \eta\left(w \otimes e_{i}^{*}\right) \otimes e_{i} \quad \text { for } w \in W
$$

where $e_{1}, \ldots, e_{n}$ is any basis for $X$ and $e_{1}^{*}, \ldots, e_{n}^{*} \in U^{*}$ any linear functions whose restrictions to $X$ give the dual basis for $X^{*}$. Let us fix $a \in A$ and check that $\theta(w a)=\theta(w) a$ for every $w \in W$. There exists a finite dimensional subspace $Z_{a} \subset H$ such that $\rho(a) \in A \otimes Z_{a}$ and $(\rho \otimes$ id $) \circ \rho(a) \in A \otimes Z_{a} \otimes Z_{a}$. Extend the chosen basis for $X$ to a basis $e_{1}, \ldots, e_{m}(m \geq n)$ for the subspace $Y=X s\left(Z_{a}\right)+X \subset U$. Lift the elements of the dual basis for $Y^{*}$ to some linear functions $e_{1}^{*}, \ldots, e_{m}^{*} \in U^{*}$. If $i>n$, then $\left.e_{i}^{*}\right|_{X}=0$, whence $\eta\left(W \otimes e_{i}^{*}\right)=0$. If $y \in Y$, then we have $\sum_{i=1}^{m}\left\langle y, e_{i}^{*}\right\rangle e_{i}=y$. In particular, this holds for elements $y$ in $X$ and in $X s\left(Z_{a}\right)$. It follows that

$$
\begin{aligned}
\sum_{(a)} \sum_{i=1}^{m} w a_{(0)} \otimes\left\langle x, e_{i}^{*} a_{(1)}\right\rangle e_{i} a_{(2)} & =\sum_{(a)} \sum_{i=1}^{m} w a_{(0)} \otimes\left\langle x s\left(a_{(1)}\right), e_{i}^{*}\right\rangle e_{i} a_{(2)} \\
& =\sum_{(a)} w a_{(0)} \otimes x s\left(a_{(1)}\right) a_{(2)} \\
& =w a \otimes x=\sum_{i=1}^{m} w a \otimes\left\langle x, e_{i}^{*}\right\rangle e_{i}
\end{aligned}
$$

in $W \otimes U$ for all $x \in X$. This shows that

$$
\sum_{(a)} \sum_{i=1}^{m} w a_{(0)} \otimes e_{i}^{*} a_{(1)} \otimes e_{i} a_{(2)}-\sum_{i=1}^{m} w a \otimes e_{i}^{*} \otimes e_{i} \in W \otimes X^{\perp} \otimes U \subset \operatorname{Ker} \eta \otimes U,
$$

and so

$$
\begin{aligned}
\theta(w a) & =\sum_{i=1}^{n} \eta\left(w a \otimes e_{i}^{*}\right) \otimes e_{i}=\sum_{i=1}^{m} \eta\left(w a \otimes e_{i}^{*}\right) \otimes e_{i} \\
& =\sum_{(a)} \sum_{i=1}^{m} \eta\left(w a_{(0)} \otimes e_{i}^{*} a_{(1)}\right) \otimes e_{i} a_{(2)} \\
& =\sum_{(a)} \sum_{i=1}^{m} \eta\left(w \otimes e_{i}^{*}\right) a_{(0)} \otimes e_{i} a_{(1)} \\
& =\sum_{i=1}^{m}\left(\eta\left(w \otimes e_{i}^{*}\right) \otimes e_{i}\right) a=\theta(w) a .
\end{aligned}
$$

Thus $\theta: W \rightarrow V \otimes U$ is a morphism in $\mathcal{M}_{A}$. 
Lemma 4.4. Let $U \in \mathcal{M}_{H}$ and $V \in \mathcal{M}_{A}$. If $V$ is an injective A-module, then so too is $V \otimes U$ provided that either $\operatorname{dim} U<\infty$ or $A$ is a right Noetherian ring, finitely generated as a right $A^{H}$-module.

Proof. We have to show that, whenever $W^{\prime}$ is a submodule of $W \in \mathcal{M}_{A}$, every morphism $\theta^{\prime}: W^{\prime} \rightarrow V \otimes U$ in $\mathcal{M}_{A}$ can be extended to a morphism $\theta: W \rightarrow V \otimes U$. Lemma 4.3 provides $\eta^{\prime}=\Phi\left(\theta^{\prime}\right)$ which is a morphism $W^{\prime} \otimes U^{*} \rightarrow V$ in $\mathcal{M}_{A}$. As $V$ is injective and $W^{\prime} \otimes U^{*}$ is an $A$-submodule of $W \otimes U^{*}$, we can extend $\eta^{\prime}$ to a morphism $\eta: W \otimes U^{*} \rightarrow V$ in $\mathcal{M}_{A}$. If $\operatorname{dim} U<\infty$, we have $\eta=\Phi(\theta)$ for some morphism $\theta: W \rightarrow V \otimes U$ in $\mathcal{M}_{A}$, and this $\theta$ extends $\theta^{\prime}$. The same argument works in general as long as we can find an extension $\eta$ vanishing on $W \otimes Y^{\perp}$ for some finite dimensional subspace $Y \subset U$.

Suppose further that $A$ is a right Noetherian ring, finitely generated as a right $A^{H}$-module. We may assume that $W^{\prime}$ is a finitely generated $A$-module. Indeed, by Baer's criterion [11, Th. 5.7.1] it suffices to consider the case where $W=A$ and $W^{\prime}$ is a right ideal of $A$. Now $W^{\prime}$ is also finitely generated as a right $A^{H}$-module. Let $w_{1}, \ldots, w_{n}$ generate $W^{\prime}$ over $A^{H}$. There exists a finite dimensional subspace $X \subset U$ such that $\theta^{\prime}\left(w_{i}\right) \in V \otimes X$ for all $i=1, \ldots, n$. As $\theta^{\prime}\left(w_{i} a\right)=\theta^{\prime}\left(w_{i}\right) \cdot(a \otimes 1)$ for $a \in A^{H}$, we have $\theta^{\prime}\left(W^{\prime}\right) \subset V \otimes X$. This implies that $\eta^{\prime}\left(W^{\prime} \otimes X^{\perp}\right)=0$. By a similar argument $\rho(A) \subset A \otimes Z$ for some finite dimensional subspace $Z \subset H$. Put $Y=X s(Z) \subset U$. Since $\rho(1)=1 \otimes 1$, we must have $1 \in Z$, and therefore $X \subset Y$. If $x \in X, \xi \in Y^{\perp}$ and $h \in Z$, then $\langle x, \xi h\rangle=\langle x s(h), \xi\rangle=0$, which shows that $Y^{\perp} Z \subset X^{\perp}$. Denote by $N \subset W \otimes U^{*}$ the $A$-submodule generated by $W \otimes Y^{\perp}$. Then

$$
N \subset\left(W \otimes Y^{\perp}\right) \cdot(A \otimes Z) \subset W \otimes X^{\perp} .
$$

Put $N^{\prime}=N \cap\left(W^{\prime} \otimes U^{*}\right)$. We see that $N^{\prime} \subset W^{\prime} \otimes X^{\perp}$, and therefore $\eta^{\prime}\left(N^{\prime}\right)=0$. Now $\left(W^{\prime} \otimes U^{*}\right) / N^{\prime}$ is embedded into $\left(W \otimes U^{*}\right) / N$ as an $A$-submodule. By injectivity of $V$, the morphism $\left(W^{\prime} \otimes U^{*}\right) / N^{\prime} \rightarrow V$ in $\mathcal{M}_{A}$ induced by $\eta^{\prime}$ extends to a morphism $\left(W \otimes U^{*}\right) / N \rightarrow V$. The latter gives the desired $\eta$.

Theorem 4.5. Let $A$ be a semilocal right Noetherian H-simple H-comodule algebra satisfying $(\mathrm{C})$. If $A$ is finitely generated as a right $A^{H}$-module, then $A$ is a quasiFrobenius ring, that is, left and right Artinian, and left and right selfinjective.

Proof. For each $V \in \mathcal{M}_{A}$ we regard $V \otimes H$ as an object of $\mathcal{M}_{A}^{H}$ letting $A$ operate via $\rho: A \rightarrow A \otimes H$ and taking id $\otimes \Delta: V \otimes H \rightarrow V \otimes H \otimes H$ as a comodule structure. If $M \in \mathcal{M}_{A}^{H}$, then $\rho_{M}: M \rightarrow M \otimes H$ is a morphism in $\mathcal{M}_{A}^{H}$ [6, Example 1.1]. In fact $\rho_{M}$ is injective as its composite with id $\otimes \varepsilon: M \otimes H \rightarrow M$ is the identity transformation of $M$. Let $E$ be an injective hull of $A$ in $\mathcal{M}_{A}$. The composite

$$
\varphi: A \stackrel{\rho}{\longrightarrow} A \otimes H \hookrightarrow E \otimes H
$$

is a monomorphism in $\mathcal{M}_{A}^{H}$. Since $(E \otimes H) / \varphi(A) \in \mathcal{M}_{A}^{H}$ is a projective $A$-module by Theorem 3.5, $\varphi$ is a split monomorphism in $\mathcal{M}_{A}$. However, $E \otimes H$ is an injective object of $\mathcal{M}_{A}$ by Lemma 4.4. Hence so too is $A$. It remains to recall that every right Noetherian right selfinjective ring is quasi-Frobenius [11, Th. 13.2.1].

\section{Coactions of finite dimensional Hopf algebras}

Let $A$ be an $H$-comodule algebra. When $H$ is finite dimensional we can improve Theorem 4.5 and derive some other conclusions. First comes an observation valid for any $H$. 
Lemma 5.1. Let $\alpha: A \rightarrow B$ be an algebra homomorphism where $B$ is any associative algebra. Consider $B \otimes H$ as an $H$-comodule algebra with respect to the comodule structure id $\otimes \Delta: B \otimes H \rightarrow B \otimes H \otimes H$. Then:

(i) $\varphi=(\alpha \otimes$ id $) \circ \rho$ is a homomorphism of $H$-comodule algebras $A \rightarrow B \otimes H$,

(ii) $\operatorname{Ker} \varphi$ is the largest $H$-costable ideal of $A$ contained in $\operatorname{Ker} \alpha$,

(iii) if $\alpha(A)=B$, then $B \otimes H=\varphi(A) \cdot(1 \otimes H)$,

(iv) if $\alpha(A)=B$ and $H$ has a bijective antipode, then $B \otimes H=(1 \otimes H) \cdot \varphi(A)$.

Proof. Clearly $\varphi$ is a composite of two algebra homomorphisms. The commutative diagram

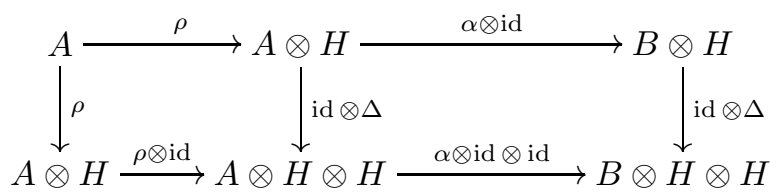

shows that $(\varphi \otimes$ id $) \circ \rho=($ id $\otimes \Delta) \circ \varphi$, i.e. $\varphi$ respects the comodule structures as well. Now $\operatorname{Ker} \varphi$ is an $H$-costable ideal of $A$. On the other hand, the composite of $\varphi$ with id $\otimes \varepsilon: B \otimes H \rightarrow B$ coincides with $\alpha$. Hence $\operatorname{Ker} \varphi \subset \operatorname{Ker} \alpha$. Conversely, $\varphi(I)=0$ whenever $I$ is an ideal of $A$ such that $\alpha(I)=0$ and $\rho(I) \subset I \otimes H$. Given $a \in A$ and $h \in H$, we have

$$
\begin{gathered}
a \otimes h=\sum_{(a)} a_{(0)} \otimes a_{(1)} s\left(a_{(2)}\right) h=\sum_{(a)} \rho\left(a_{(0)}\right) \cdot\left(1 \otimes s\left(a_{(1)}\right) h\right) \in \rho(A) \cdot(1 \otimes H), \\
a \otimes h=\sum_{(a)} a_{(0)} \otimes h s^{-1}\left(a_{(2)}\right) a_{(1)}=\sum_{(a)}\left(1 \otimes h s^{-1}\left(a_{(1)}\right)\right) \cdot \rho\left(a_{(0)}\right) \in(1 \otimes H) \cdot \rho(A)
\end{gathered}
$$

in $A \otimes H$. Applying the homomorphism $\alpha \otimes$ id : $A \otimes H \rightarrow B \otimes H$, we deduce (iii) and (iv).

Part (ii) of the next result can be viewed as a generalization of the fact proved in 18 according to which all Frobenius right coideal subalgebras of a semisimple Hopf algebra are themselves semisimple. Corollary 5.3 is close in spirit to Linchenko's result [15] which shows that, under restrictions on char $k$, the Jacobson radical of a finite dimensional $H$-module algebra for an involutory Hopf algebra is $H$-stable. Extension of the latter work to polynomial identity module algebras appeared in [16. It will be assumed in the rest of this section that $\operatorname{dim} H<\infty$.

Theorem 5.2. If $A$ is right Noetherian and there exists $P \in$ Max $A$ such that $A / P$ is left Artinian and $P$ contains no nonzero $H$-costable ideals of $A$, then:

(i) $A$ is $H$-simple and is a quasi-Frobenius ring,

(ii) $A$ is semisimple Artinian provided that so is $H$.

Proof. Define the algebra homomorphism $\varphi: A \rightarrow A / P \otimes H$ taking $B=A / P$ and $\alpha: A \rightarrow B$ as the canonical homomorphism in Lemma 5.1. Then $\operatorname{Ker} \varphi$ is an $H$-costable ideal of $A$ contained in $P$. Hence $\operatorname{Ker} \varphi=0$ by the hypotheses.

We will regard $M=A / P \otimes H$ as an $(A / P, A)$-bimodule letting $A$ and $A / P$ operate on $M$ via $\varphi$ and the canonical isomorphism $A / P \rightarrow A / P \otimes 1$, respectively. Since the ring $A / P$ is left Artinian, it has finite length as a left module over itself (e.g., [11, Cor. 9.3.12]). Then so too does the finitely generated left $A / P$-module $M$. By Lemma $5.1 M$ is a finitely generated right $A$-module (as the antipodes of finite dimensional Hopf algebras are bijective). The latter module is therefore 
Noetherian. Applying Lenagan's Theorem [23, Th. 4.1.6] with left and right sides interchanged, we conclude that the right $A$-module $M$ has finite length. Since $\varphi$ is injective, $A$ has to be right Artinian. Then $A$ is semilocal [11, Cor. 9.2.3]. For any ideal $I$ of $A$ the ring $A / I \otimes H$ is right Artinian, as it is finitely generated as a right module over $A / I \otimes 1$. Therefore $A$ satisfies (C). Proposition 3.7 shows that $A$ is $H$-simple. To complete the proof of (i) we can proceed as in Theorem 4.5 (Lemma 4.4 still works).

As in Theorem 4.5 we have $V \otimes H \in \mathcal{M}_{A}^{H}$, and so $V \otimes H$ is a projective $A$ module, for any $V \in \mathcal{M}_{A}$. Suppose that $H$ is semisimple. Then any cyclic right $H$-module $U$ is a direct summand of $H$ in $\mathcal{M}_{H}$, whence $V \otimes U$ is a direct summand of $V \otimes H$ in $\mathcal{M}_{A}$. Taking $U=k$, we see that $V \cong V \otimes k$ is a direct summand of a free $A$-module. In other words, all right $A$-modules are projective, whence (ii).

Corollary 5.3. Denote $J=\bigcap_{P \in \mathcal{F}} P$ where $\mathcal{F}$ is the set of all maximal ideals $P$ of $A$ such that the factor ring $A / P$ is left Artinian. If $A$ is right Noetherian and $H$ is semisimple, then $J$ is an $H$-costable ideal of $A$.

Proof. For each $P \in \mathcal{F}$ denote by $I_{P}$ the largest $H$-costable ideal of $A$ contained in $P$. The $H$-comodule algebra $A / I_{P}$ has no nonzero $H$-costable ideals contained in $P / I_{P}$. Theorem 5.2(ii) then shows that $A / I_{P}$ is semisimple Artinian. In particular, $I_{P}$ coincides with the intersection of those $Q \in \operatorname{Max} A$ for which $I_{P} \subset Q$. For each $Q$ appearing here the factor ring $A / Q$ is simple Artinian so that $Q \in \mathcal{F}$. Hence $J \subset I_{P} \subset P$ for each $P$. It follows that $J=\bigcap_{P \in \mathcal{F}} I_{P}$, and we are done.

Proposition 5.4. Suppose that $A$ is $H$-simple and $\operatorname{dim} A<\infty$. Let $V$ be a finite dimensional and $W$ a simple right $A$-module. Denoting $D=\operatorname{End}_{A} W$, we have

$$
(\operatorname{dim} D)(\operatorname{dim} A) \mid(\operatorname{dim} V)(\operatorname{dim} W)(\operatorname{dim} H) .
$$

Proof. Under present hypotheses $D$ is a skew field and $W$ a finite dimensional vector space over $D$. Let $t=\operatorname{dim}_{D} W$, and let $Q \in \operatorname{Max} A$ be the annihilator of $W$ in $A$. Note that $A / Q$ is a simple Artinian ring such that $A / Q \cong W^{t}$ in $\mathcal{M}_{A}$. As before $M=V \otimes H$ may be regarded as an object of $\mathcal{M}_{A}^{H}$. The $A / Q$-module $(M / M Q)^{t}$ is free since its length is divisible by $t$. Theorem 3.5 shows that $M^{t}$ is a free $A$-module, whence $\operatorname{dim} A$ divides $t(\operatorname{dim} M)$. It remains to observe that $(\operatorname{dim} D) t=\operatorname{dim} W$ and $\operatorname{dim} M=(\operatorname{dim} V)(\operatorname{dim} H)$.

The previous result was proved in [42, Th. 2.2] under the additional assumptions that $k$ is algebraically closed of characteristic zero, $H$ is semisimple, $A^{H}=k$ and $V=W$.

\section{Finite dimensional coideal subalgebras}

Let $A \subset H$ be a right coideal subalgebra. The opposite multiplication in $H$ and the same comultiplication produce a bialgebra $H^{\mathrm{op}}$ which contains $A^{\mathrm{op}}$ as a right coideal subalgebra. All conclusions of the next result do not change when the pair $A, H$ is replaced with $A^{\mathrm{op}}, H^{\mathrm{op}}$. Consequently, Theorem 6.1 is valid not only when $H$ is a Hopf algebra but also when $H$ is a bialgebra for which $H^{\mathrm{op}}$ has an antipode. Bialgebras with the latter property appeared in the literature under the name of anti-Hopf algebras. The same extension applies to Theorem 4.2. 
Theorem 6.1. Let $H$ be a weakly finite (anti-)Hopf algebra and $A \subset H$ a finite dimensional right coideal subalgebra. Denote $D=H / H A^{+}$and $D^{\prime}=H / A^{+} H$ where $A^{+}=\left.\operatorname{Ker} \varepsilon\right|_{A}$. Then:

(i) $A$ is Frobenius and is a simple object of both $\mathcal{M}_{A}^{H}$ and ${ }_{A} \mathcal{M}^{H}$,

(ii) all objects of both $\mathcal{M}_{A}^{H}$ and ${ }_{A} \mathcal{M}^{H}$ are free $A$-modules,

(iii) there are canonical category equivalences $\mathcal{M}_{A}^{H} \approx \mathcal{M}^{D}$ and ${ }_{A} \mathcal{M}^{H} \approx \mathcal{M}^{D^{\prime}}$,

(iv) there exist isomorphisms $H \cong D \otimes A$ in ${ }^{D} \mathcal{M}_{A}$ and $H \cong A \otimes D^{\prime}$ in ${ }_{A}^{D^{\prime} \mathcal{M}}$.

Proof. Recall that $H$ is a simple object of $\mathcal{M}_{H}^{H}$. If $I$ is an $H$-costable ideal of $A$, then $I H$ is an $\mathcal{M}_{H}^{H}$-subobject of $H$, so that $I H$ is either 0 or $H$. If, in addition, $I \subset A^{+}$, then $\varepsilon(I H)=0$, whence $I H=0$. We conclude that $A^{+}$contains no nonzero $H$ costable ideals of $A$. Proposition 3.7 shows that $A$ is $H$-simple. Theorem 4.2 gives the first part of (i) and also (ii) since $A / A^{+} \cong k$. Furthermore, $A$ is a simple object of $\mathcal{M}_{A}^{H}$ by Corollary 3.6. A similar argument works for ${ }_{A} \mathcal{M}^{H}$.

Now $H \in{ }_{A} \mathcal{M}^{H}$ is a free left $A$-module, and the first equivalence in (iii) is obtained by an application of [41, Th. 1]. If $H$ has a bijective antipode $s$, then $H^{\mathrm{op}}$ is again a Hopf algebra. As $H^{\mathrm{op}}$ is weakly finite, $H^{\mathrm{op}}$ and $A^{\mathrm{op}}$ meet the hypotheses of Theorem 6.1. The second equivalence in (iii) is then also fulfilled because ${ }_{A} \mathcal{M}^{H}=\mathcal{M}_{A^{\text {op }}}^{\text {op }}$ [7, (1.2)]. To prove (iii), not assuming the bijectivity of $s$ requires a different argument which will be provided in Lemma 6.2. A selfcontained proof of (iv) will be offered in Lemma 6.4; it streamlines the arguments already known. Lemma 6.4 can be applied with $M=H$ since $H \in{ }_{A}^{D^{\prime} \mathcal{M}}{ }^{H}$ and $H \in{ }^{D} \mathcal{M}_{A}^{H}$.

Remarks. For a finite dimensional $H$ it was shown by Masuoka [18] that (ii) and (iv) are each equivalent to $A$ being Frobenius, among other equivalent conditions.

In the case of a commutative $H$ the equivalence $\mathcal{M}_{A}^{H} \approx \mathcal{M}^{D}$ admits an interpretation in terms of Mackey imprimitivity theory for algebraic groups and group schemes [3], 30. The imprimitivity theorem of Koppinen and Neuvonen [12] for arbitrary finite dimensional Hopf algebras can be put into this context as well.

Isomorphisms of (iv) were christened the normal basis property. Initially this property was studied in connection with the structure of Hopf Galois extensions (e.g., 14]). Some conditions ensuring its fulfillment were given by Schneider 35. In particular, [35, Th. 2.4] yields (iv) in the case where $\operatorname{dim} H<\infty$ and $A$ is a Hopf subalgebra.

According to [21, Prop. 3.2] the first isomorphism in (iv) is equivalent to the $A$ cocleftness of $H$, that is, to the existence of a morphism $H \rightarrow A$ in $\mathcal{M}_{A}$ invertible in the convolution algebra $\operatorname{Hom}(H, A)$. By [21, Th. 3.4] $H$ is $A$-cocleft provided that $H$ is a faithfully coflat left $D$-comodule and all objects of $\mathcal{M}_{L \otimes A}^{L \otimes H}$ are free $L \otimes A$-modules where $L$ is an algebraic closure of $k$. The first of the two hypotheses here means that the cotensor product functor ? $\square_{D} H$ is faithfully exact, which is a consequence of the first equivalence in (iii). Since $L \otimes A$ is a finite dimensional right coideal subalgebra of the Hopf algebra $L \otimes H$ over $L$, the freeness in $\mathcal{M}_{L \otimes A}^{L \otimes H}$ is also fulfilled by (i). This proves a part of (iv) already at this stage.

Suppose that char $k \neq 2$. The Hopf algebra described in 28, call it $H$, contains a grouplike element $g$ and a $3 \times 3$ matrix coalgebra $C$ with a basis $c_{i j}(1 \leq i, j \leq 3)$ 
such that

$$
g^{2}=1, \quad g c_{i j}=\lambda_{i} \lambda_{j} c_{i j}, \quad \Delta\left(c_{i j}\right)=\sum_{l=1}^{3} c_{i l} \otimes c_{l j}, \quad \varepsilon\left(c_{i j}\right)=\delta_{i j}
$$

where $\lambda_{1}=\lambda_{2}=1$ and $\lambda_{3}=-1$. In this example $C$ is an ${ }_{A} \mathcal{M}^{H}$-subobject of $H$ but $C$ is not a free left $A$-module. Note that $s\left(c_{l t}\right) g=s\left(g c_{l t}\right)=\lambda_{l} \lambda_{t} s\left(c_{l t}\right)$ for $1 \leq l, t \leq 3$. Since

$$
\lambda_{i} \lambda_{j} s\left(c_{l t}\right) c_{i j}=s\left(c_{l t}\right) g c_{i j}=\lambda_{l} \lambda_{t} s\left(c_{l t}\right) c_{i j},
$$

we have $s\left(c_{l t}\right) c_{i j}=0$ whenever $\lambda_{i} \lambda_{j} \neq \lambda_{l} \lambda_{t}$. Using these equalities together with the identities $\sum_{i} s\left(c_{l i}\right) c_{i j}=\delta_{l j}$ it is easy to see that the matrices

$$
X=\left[\begin{array}{ll}
s\left(c_{11}+c_{13}\right) & s\left(c_{12}\right) \\
s\left(c_{21}+c_{23}\right) & s\left(c_{22}\right)
\end{array}\right], \quad Y=\left[\begin{array}{cc}
c_{11}+c_{31} & c_{12}+c_{32} \\
c_{21} & c_{22}
\end{array}\right], \quad Z=\left[\begin{array}{cc}
0 & 0 \\
c_{23} & 0
\end{array}\right]
$$

fulfill the equations $X Y=1$ and $X Z=0$ in $\operatorname{Mat}_{2}(H)$. Thus $X$ has a right inverse but no left inverse, and so $H$ is not weakly 2 -finite.

Lemma 6.2. Let $H$ be any bialgebra, $A$ its right coideal subalgebra and $D=$ $H / H A^{+}, D^{\prime}=H / A^{+} H$ quotient coalgebras. Define a functor

$$
\Phi: \mathcal{M}_{A}^{H} \rightsquigarrow \mathcal{M}^{D} \quad\left(\text { resp. }, \Phi:{ }_{A} \mathcal{M}^{H} \rightsquigarrow \mathcal{M}^{D^{\prime}}\right)
$$

by $M \mapsto M / M A^{+}$(resp., $\left.M \mapsto M / A^{+} M\right)$. If $\Phi$ is faithfully exact, then it is an equivalence. In particular, this is the case whenever all nonzero objects of $\mathcal{M}_{A}^{H}$ (resp., ${ }_{A} \mathcal{M}^{H}$ ) are projective generators in $\mathcal{M}_{A}$ (resp., $\left.{ }_{A} \mathcal{M}\right)$.

The equivalence $\mathcal{M}_{A}^{H} \approx \mathcal{M}^{D}$ was verified in [41, Th. 1] under the hypothesis that some $N \in{ }_{H} \mathcal{M}$ is faithfully flat as a left $A$-module. If $N$ is such a module, then the faithful exactness of $\Phi$ follows from the isomorphisms $M \otimes_{A} N \cong \Phi(M) \otimes N$ constructed in [41] for all $M \in \mathcal{M}_{A}^{H}$. However, the proof of the equivalence given there does not carry over to our present situation. We will treat ${ }_{A} \mathcal{M}^{H}$, thereby completing the proof of Theorem 6.1(iii). The other case is similar, as we may replace $A, H$ with $A^{\text {op }}, H^{\mathrm{op}}$.

Proof. The map $\lambda: H \stackrel{\Delta}{\longrightarrow} H \otimes H \stackrel{\text { can } \otimes \mathrm{id}}{\longrightarrow} D^{\prime} \otimes H$ makes $H$ into a left $D^{\prime}$-comodule. Now $H$ is an object of ${ }_{A} \mathcal{M}^{H}$, and $\lambda$ commutes with left multiplications by elements of $A$ and the right $H$-comodule structure on $H$. For each vector space $V$ we regard $V \otimes H$ as an object of ${ }_{A} \mathcal{M}^{H}$ using available operations on the second tensorand. Define a functor $\Psi: \mathcal{M}^{D^{\prime}} \rightsquigarrow{ }_{A} \mathcal{M}^{H}$ by the rule

$$
\Psi(V)=V \square_{D^{\prime}} H=\operatorname{Ker}\left(V \otimes H \stackrel{\text { id } \otimes \lambda-\mu \otimes \mathrm{id}}{\longrightarrow} V \otimes D^{\prime} \otimes H\right)
$$

where $\mu: V \rightarrow V \otimes D^{\prime}$ is the $D^{\prime}$-comodule structure on $V \in \mathcal{M}^{D^{\prime}}$ (basic properties of cotensor products are summarized in [40, Appendix 2]; details can be found in 4, Ch. 2]). It is immediate that $\Psi(V)$ is an ${ }_{A} \mathcal{M}^{H}$-subobject of $V \otimes H$. For $M \in{ }_{A} \mathcal{M}^{H}$ and $V \in \mathcal{M}^{D^{\prime}}$ there are natural morphisms

$$
\Xi_{M}: M \rightarrow \Psi \Phi(M), \quad \Theta_{V}: \Phi \Psi(V) \rightarrow V .
$$

Here $\Xi_{M}$ coincides with the composite $M \stackrel{\rho}{\longrightarrow} M \otimes H \stackrel{\text { can } \otimes \text { id }}{\longrightarrow} \Phi(M) \otimes H$; one checks that $\Xi_{M}$ takes values in $\Phi(M) \square_{D^{\prime}} H$. The map id $\otimes \varepsilon: V \otimes H \rightarrow V$ vanishes on $V \otimes A^{+} H$, and so the restriction of this map to $\Psi(V)$ factors through $\Phi \Psi(V)$; one takes $\Theta_{V}$ to be the induced map. 
We have $\Psi\left(D^{\prime}\right) \cong H$ by [40. To be precise, $\lambda$ maps $H$ isomorphically onto $\Psi\left(D^{\prime}\right) \subset D^{\prime} \otimes H$. Since (id $\left.\otimes \varepsilon\right) \circ \lambda$ coincides with the canonical projection $H \rightarrow D^{\prime}$, we can identify $\Theta_{D^{\prime}}$ with the identity map $H / A^{+} H \rightarrow D^{\prime}$. In other words, $\Theta_{D^{\prime}}$ is an isomorphism. It is immediate from the definitions that both $\Phi$ and $\Psi$ commute with arbitrary direct sums. Therefore $\Theta_{E}$ is an isomorphism whenever $E \in \mathcal{M}^{D^{\prime}}$ is a direct sum of an arbitrary family of copies of $D^{\prime}$. Now $D^{\prime}$ is an injective cogenerator in $\mathcal{M}^{D^{\prime}}$ [4, Prop. 2.4.3 and Cor. 2.4.5]. This implies that each $V \in \mathcal{M}^{D^{\prime}}$ is the kernel of a morphism $E \rightarrow E^{\prime}$ in $\mathcal{M}^{D^{\prime}}$ where both $E$ and $E^{\prime}$ are direct sums of copies of $D^{\prime}$. In the commutative diagram

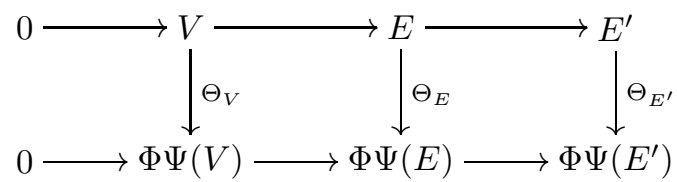

both $\Theta_{E}$ and $\Theta_{E^{\prime}}$ are isomorphisms. By 40 the cotensor products are left exact. In particular, $\Psi$ is left exact. Since $\Phi$ is exact, the bottom row in the diagram above is exact. It follows that $\Theta_{V}$ is an isomorphism for any $V$.

Now let $M \in{ }_{A} \mathcal{M}^{H}$ and $V=\Phi(M)$. Denote by $K$ and $L$ the kernel and the cokernel of $\Xi_{M}$. By the exactness of $\Phi$ we have an exact sequence

$$
0 \longrightarrow \Phi(K) \longrightarrow \Phi(M) \stackrel{\Phi\left(\Xi_{M}\right)}{\longrightarrow} \Phi \Psi(V) \longrightarrow \Phi(L) \longrightarrow 0 .
$$

The composite of $\Phi\left(\Xi_{M}\right)$ with $\Theta_{V}$ is just the identity map $\Phi(M) \rightarrow V$. Since $\Theta_{V}$ is an isomorphism, so is $\Phi\left(\Xi_{M}\right)$ as well. We deduce that $\Phi(K)=0$ and $\Phi(L)=0$. Then $K=0$ and $L=0$ by faithfulness of $\Phi$. Thus $\Xi_{M}$ is an isomorphism for any $M$ as well.

If all objects of ${ }_{A} \mathcal{M}^{H}$ are projective in ${ }_{A} \mathcal{M}$, then all exact sequences in ${ }_{A} \mathcal{M}^{H}$ split in ${ }_{A} \mathcal{M}$, whence $\Phi$ is exact. If $M \in{ }_{A} \mathcal{M}^{H}$ is a generator in ${ }_{A} \mathcal{M}$, then $A^{+} M \neq$ $M$ so that $\Phi(M) \neq 0$. This completes the proof.

Lemma 6.3. Let $D$ be a coalgebra and $M \in{ }^{D} \mathcal{M}$. For each subcoalgebra $C \subset D$ put $M_{C}=\{m \in M \mid \lambda(m) \in C \otimes M\}$ where $\lambda: M \rightarrow D \otimes M$ is the given comodule structure. Then $M \cong D^{n}$ for some fixed $n \geq 0$ if and only if $\operatorname{dim} M_{C}=n \operatorname{dim} C$ for each finite dimensional subcoalgebra $C$.

Proof. Let $\Delta: D \rightarrow D \otimes D$ be the comultiplication and $\varepsilon: D \rightarrow k$ the counit. If $v \in D$ satisfies $\Delta(v) \in C \otimes D$, then $v=(\operatorname{id} \otimes \varepsilon) \circ \Delta(v) \in C$. Hence $M_{C} \cong C^{n}$ whenever $M \cong D^{n}$. This proves one direction of the lemma. Conversely, suppose $\operatorname{dim} M_{C}=n \operatorname{dim} C$ for each finite dimensional $C$. If $S \subset D$ is a simple subcoalgebra and $V$ is a simple left $S$-comodule, then $S \cong V^{t}$ in ${ }^{S} \mathcal{M}$ where $t=\operatorname{dim} S / \operatorname{dim} V$. It then follows that $M_{S} \cong V^{n t} \cong S^{n}$. The socle of the $D$-comodule $M$ coincides with the sum $\sum M_{S}$ over all simple subcoalgebras $S \subset D$. Hence soc $M \cong \bigoplus S^{n} \cong$ soc $D^{n}$ in $D \mathcal{M}$. Note that $D^{n}$ is an injective $D$-comodule [4, Cor. 2.4.5]. Any embedding $\operatorname{soc} M \rightarrow D^{n}$ therefore extends to a morphism $\psi: M \rightarrow D^{n}$ in ${ }^{D} \mathcal{M}$. In fact $\psi$ is injective since so is its restriction to the socle. One has $\psi\left(M_{C}\right) \subset C^{n}$ for each subcoalgebra $C$. Comparing the dimensions, we deduce that $\psi\left(M_{C}\right)=C^{n}$ whenever $\operatorname{dim} C<\infty$. As $D$ is a union of finite dimensional subcoalgebras, $\psi$ is surjective.

Let ${ }^{D} \mathcal{M}_{A}^{H}$ be the category whose objects are vector spaces together with a left $D$-comodule, a right $H$-comodule and a right $A$-module structures, such that the 
$H$-comodule and $A$-module structures satisfy the compatibility condition required in $\mathcal{M}_{A}^{H}$ and these two structures commute with the $D$-comodule structure. The category ${ }_{A}^{D} \mathcal{M}^{H}$ is defined similarly with ${ }_{A} \mathcal{M}^{H}$ in place of $\mathcal{M}_{A}^{H}$. For $V \in{ }^{D} \mathcal{M}$ we regard $V \otimes A$ as an object of either ${ }^{D} \mathcal{M}_{A}$ or ${ }_{A}^{D} \mathcal{M}$ so that $A$ operates by multiplications on the second tensorand and the comodule structure comes from that on $V$.

Lemma 6.4. Let $A, H$ be as in Theorem 6.1 and $D$ any coalgebra. If $M \in{ }^{D} \mathcal{M}_{A}^{H}$ (resp. $\left.M \in{ }_{A}^{D} \mathcal{M}^{H}\right)$ and $M / M A^{+} \cong D\left(\right.$ resp. $\left.M / A^{+} M \cong D\right)$ in ${ }^{D} \mathcal{M}$, then $M \cong$ $D \otimes A$ in ${ }^{D} \mathcal{M}_{A}\left(\right.$ resp. in $\left.{ }_{A}^{D} \mathcal{M}\right)$.

Proof. We consider only ${ }^{D} \mathcal{M}_{A}^{H}$. Since $A$ is Frobenius, there exists $0 \neq x \in A$ such that $A^{+} x=0$. For each subcoalgebra $C \subset D$ define $M_{C}$ as in Lemma 6.3. Since the $D$-comodule structure on $M$ commutes with the two other structures, $M_{C}$ is an $\mathcal{M}_{A}^{H}$-subobject of $M$. By Theorem 6.1 $M_{C}$ is a free $A$-module. Then the annihilator of $x$ in $M_{C}$ coincides with $M_{C} A^{+}$, and so the action of $x$ induces an isomorphism $M_{C} / M_{C} A^{+} \cong M_{C} x$ in ${ }^{C} \mathcal{M}$. Taking $C=D$, we obtain an isomorphism $M x \cong D$ in ${ }^{D} \mathcal{M}$. Next, $M_{C}$ is an $A$-module direct summand of $M$ since $M / M_{C} \in \mathcal{M}_{A}^{H}$ is a free $A$-module. It follows that $M_{C} x=M_{C} \cap M x \cong C$. If $\operatorname{dim} C<\infty$, then $\operatorname{dim} M_{C} / M_{C} A^{+}=\operatorname{dim} C$, and this number is equal to the rank of the free $A$-module $M_{C}$, so that $\operatorname{dim} M_{C}=(\operatorname{dim} C)(\operatorname{dim} A)$. By Lemma $6.3 M \cong D^{n}$ in ${ }^{D} \mathcal{M}$ where $n=\operatorname{dim} A$.

Suppose that $S \subset D$ is a simple subcoalgebra and $R=S^{*}$ the dual simple algebra. By Corollary $3.8 B=R \otimes A$ is an $H$-simple $H$-comodule algebra with a maximal ideal $P=R \otimes A^{+}$. The $S$-comodule structure on objects of ${ }^{S} \mathcal{M}_{A}^{H}$ corresponds to an $R$-module structure commuting with the $A$-module and $H$-comodule structures. In other words, ${ }^{S} \mathcal{M}_{A}^{H} \cong \mathcal{M}_{B}^{H}$. Now we have $M_{S} \in \mathcal{M}_{B}^{H}$. As we have seen, $M_{S} / M_{S} P=M_{S} / M_{S} A^{+} \cong S$ in ${ }^{S} \mathcal{M} \approx \mathcal{M}_{R}$. Every simple finite dimensional algebra is Frobenius. Hence $S$ is a free $R$-module of rank 1. By Theorem $4.2 M_{S}$ is a free $B$-module of rank 1 . There then exists an $R$-submodule, i.e. an $S$-subcomodule, $U_{S} \subset M_{S}$ such that the map $U_{S} \otimes A \rightarrow M_{S}$ afforded by the $A$-module structure is bijective.

We choose such a subcomodule $U_{S}$ for each simple subcoalgebra $S \subset D$ and put $U=\sum_{S} U_{S}$. Let $V$ be a maximal $D$-subcomodule of $M$ containing $U$ as an essential subcomodule (so that $U \cap W \neq 0$ for every $D$-subcomodule $0 \neq W \subset V$ ). Since $M \cong D^{n}$ is an injective $D$-comodule, $V$ is a direct summand of $M$ in ${ }^{D} \mathcal{M}$. In particular, $V$ is an injective $D$-comodule. The canonical map $\varphi: V \otimes A \rightarrow M$ is a morphism in ${ }^{D} \mathcal{M}_{A}$. By the choice of $U_{S}$ the restriction of $\varphi$ to $U_{S} \otimes A$ is a bijection onto $M_{S}$ for each $S$. The sum $\sum_{S} M_{S}$ over all simple subcoalgebras is direct since the $M_{S}$ 's are the isotypic components of the socle $\operatorname{soc}^{D} M$ of the $D$-comodule $M$. Hence the restriction of $\varphi$ to $U \otimes A$ is injective. As $U \otimes A$ is an essential subcomodule of $V \otimes A$, we see that $\varphi$ itself is injective. Now $V \otimes A$ is a direct sum of copies of $V$ in ${ }^{D} \mathcal{M}$. It is therefore an injective $D$-comodule, whence $\operatorname{Im} \varphi$ is a direct summand of $M$ in ${ }^{D} \mathcal{M}$. On the other hand, the inclusion $\operatorname{soc}^{D} M \subset \operatorname{Im} \varphi$ entails the surjectivity of $\varphi$. Thus $\varphi$ is an isomorphism. It follows also that $V \cong M / M A^{+}$in ${ }^{D} \mathcal{M}$, and we are done.

Remark. According to [34, Cor. 2.2] (with left and right sides interchanged) an object $M \in{ }^{D} \mathcal{M}_{A}$ is isomorphic to $D \otimes A$ provided that the following two conditions are fulfilled: $M$ is injective in ${ }^{D} \mathcal{M}$ and $S \square_{D} M \cong S \otimes A$ in ${ }^{S} \mathcal{M}_{A}$ for each simple 
subcoalgebra $S \subset D$. The verification of these conditions were two main steps in the proof of Lemma 6.4 (note that $S \square_{D} M \cong M_{S}$ ).

Theorem 6.1 enables us to strengthen [18, Prop. 2.10]:

Corollary 6.5. If $H$ is a finite dimensional Hopf algebra, then there is a bijective correspondence between the right coideal subalgebras in $H$ and $H^{*}$.

\section{Dualization to module algebras}

The results of section 3 have their counterparts for $H$-module algebras $A$. In fact condition (C) on comodule algebras is no longer needed. The reason is that the weak finiteness of convolution algebras is recognized by the algebra argument alone. If $A$ is semilocal, then so are all its factor rings $A / I$, which are therefore weakly finite.

Lemma 7.1. If $B$ is a weakly finite algebra and $C$ any coalgebra, then the convolution algebra $\operatorname{Hom}(C, B)$ is weakly finite.

Proof. Note that $\operatorname{Mat}_{n}(\operatorname{Hom}(C, B)) \cong \operatorname{Hom}\left(C, B_{n}\right)$ where $B_{n}=\operatorname{Mat}_{n}(B)$. Two linear functions $C \rightarrow B_{n}$ coincide if and only if they have the same restriction to every finite dimensional subcoalgebra of $C$. It therefore suffices to prove that the convolution algebra $\operatorname{Hom}\left(C, B_{n}\right)$ is weakly 1-finite under the assumption that $\operatorname{dim} C<\infty$. In this case $C^{*}$ is a finite dimensional algebra and $\operatorname{Hom}\left(C, B_{n}\right) \cong$ $B_{n} \otimes C^{*}$. The ring $B_{n}$ is a free module of finite rank over its subring isomorphic to $B$. The same is then valid for $B_{n} \otimes C^{*}$, and we may apply Proposition $2.2(\mathrm{e})$.

Let $A$ be an $H$-module algebra and $M \in{ }_{H} \mathcal{M}_{A}$. Define $\hat{m} \in \operatorname{Hom}(H, M)$ for each $m \in M$ by the rule $\hat{m}(h)=\varepsilon(h) m$ for $h \in H$. We then have $(\hat{m} \eta)(h)=m \eta(h)$ for $\eta \in \operatorname{Hom}(H, A)$ and $h \in H$. In Lemmas $7.2,7.3,7.4$ we assume that $e_{1}, \ldots, e_{n}$ generate $M$ as an $A$-module.

Lemma 7.2. Under previous assumptions $\hat{e}_{1}, \ldots, \hat{e}_{n}$ and $\tau\left(e_{1}\right), \ldots, \tau\left(e_{n}\right)$ are two systems of generators for the $\operatorname{Hom}(H, A)$-module $\operatorname{Hom}(H, M)$.

Proof. Let $\xi \in \operatorname{Hom}(H, M)$. There exist $\eta_{1}, \ldots, \eta_{n} \in \operatorname{Hom}(H, A)$ such that $\xi(h)=$ $\sum_{i=1}^{n} e_{i} \eta_{i}(h)$ for all $h \in H$, and it follows that $\xi=\sum_{i=1}^{n} \hat{e}_{i} \eta_{i}$. Now take $m \in M$ and define $\xi$ by the rule $\xi(h)=s(h) m$. We get

$$
\begin{aligned}
\varepsilon(h) m & =\sum_{(h)} h_{(1)} s\left(h_{(2)}\right) m=\sum_{(h)} h_{(1)}\left(\sum_{i=1}^{n} e_{i} \eta_{i}\left(h_{(2)}\right)\right) \\
& =\sum_{i=1}^{n} \sum_{(h)}\left(h_{(1)} e_{i}\right)\left(h_{(2)} \eta_{i}\left(h_{(3)}\right)\right)=\sum_{i=1}^{n} \sum_{(h)}\left(h_{(1)} e_{i}\right) \theta_{i}\left(h_{(2)}\right)
\end{aligned}
$$

where $\theta_{i} \in \operatorname{Hom}(H, A)$ is defined by the rule $\theta_{i}(h)=\sum_{(h)} h_{(1)} \eta_{i}\left(h_{(2)}\right)$. This shows that $\hat{m}=\sum_{i=1}^{n} \tau\left(e_{i}\right) \theta_{i}$, and we have already checked that $\hat{e}_{1}, \ldots, \hat{e}_{n}$ generate $\operatorname{Hom}(H, M)$.

Lemma 7.3. Suppose that $I$ is an ideal of $A$ such that $\mathcal{I}_{e_{1}, \ldots, e_{n}} \subset I$ and the ring $A / I$ is weakly finite. Then there exists an $H$-stable ideal $K$ of $A$ such that $\mathcal{I}_{e_{1}, \ldots, e_{n}} \subset K \subset I$. 
Proof. Note that $\operatorname{Hom}(H, I)$ is an ideal of $\operatorname{Hom}(H, A)$, and the factor algebra by this ideal is isomorphic to $\operatorname{Hom}(H, A / I)$. This factor algebra is weakly finite by Lemma 7.1. Denote by $K$ the preimage of $\operatorname{Hom}(H, I)$ under $\tau: A \rightarrow \operatorname{Hom}(H, A)$. Clearly $K=\{a \in A \mid H a \subset I\}$ so that $K$ is an $H$-stable ideal of $A$ contained in $I$.

If $\eta_{1}, \ldots, \eta_{n} \in \operatorname{Hom}(H, A)$ are such that $\sum_{i=1}^{n} \hat{e}_{i} \eta_{i}=0$, then $\sum_{i=1}^{n} e_{i} \eta_{i}(h)=0$ for all $h \in H$, and so $\eta_{i}(H) \subset I$ for all $i=1, \ldots, n$. It follows that $\mathcal{I}_{\hat{e}_{1}, \ldots, \hat{e}_{n}}$ is contained in $\operatorname{Hom}(H, I)$. By Lemma $2.3 \mathcal{I}_{\tau\left(e_{1}\right), \ldots, \tau\left(e_{n}\right)} \subset \operatorname{Hom}(H, I)$ as well. Suppose that $\sum_{i=1}^{n} e_{i} x_{i}=0$ for some $x_{1}, \ldots, x_{n} \in A$. Then $\sum_{i=1}^{n} \tau\left(e_{i}\right) \tau\left(x_{i}\right)=0$ in $\operatorname{Hom}(H, M)$, which shows that $\tau\left(x_{i}\right) \in \mathcal{I}_{\tau\left(e_{1}\right), \ldots, \tau\left(e_{n}\right)}$, yielding the inclusions $x_{i} \in K$ for all $i=1, \ldots, n$. This means that $\mathcal{I}_{e_{1}, \ldots, e_{n}} \subset K$.

The proofs of Lemmas 3.3, 3.4 and Theorem 3.5 generalize without further complications to the case of module algebras:

Lemma 7.4. Suppose that $I$ is an ideal of $A$ such that $A / I$ is weakly finite and $I$ contains no nonzero $H$-stable ideals of $A$. If the cosets of $e_{1}, \ldots, e_{n}$ give a basis for the $A / I$-module $M / M I$, then $e_{1}, \ldots, e_{n}$ are a basis for the $A$-module $M$.

Lemma 7.5. Let $A$ be a semilocal H-module algebra. Suppose that $M$ is A-finite and there exists $P \in$ Max $A$ such that $P$ contains no nonzero $H$-stable ideals of $A$ and $r_{P}(M) \geq r_{Q}(M)$ for all $Q \in \operatorname{Max} A$ (in the notations of Lemma 3.4). Then the conclusions (i) and (ii) of Lemma 3.4 are fulfilled.

Theorem 7.6. Suppose that $A$ is a semilocal H-simple H-module algebra, and let $M \in{ }_{H} \mathcal{M}_{A}$ be locally $A$-finite. Then $M$ is a projective $A$-module. Moreover, $M$ is a free $A$-module if and only if $M / M Q$ is a free $A / Q$-module for at least one $Q \in \operatorname{Max} A$.

The reader may wish to reformulate this result in terms of smash product algebras. Here $H^{\text {cop }}$ rather than $H$ has to be a Hopf algebra:

Corollary 7.7. Let $H$ be an anti-Hopf algebra and $A$ a semilocal $H$-simple $H$ module algebra. Then all locally $A$-finite left $A \# H$-modules are projective $A$-modules.

If an $H$-module algebra $A$ is not $H$-simple, one can ask about projectivity of localizations. To be precise, let $A$ be right Noetherian, and let $P$ be a semiprime ideal of $A$. Denote by $\mathcal{C}_{A}(P) \subset A$ the preimage of the set of regular elements in $A / P$. One says that $P$ is right localizable if $\mathcal{C}_{A}(P)$ is a right denominator set [23. Ch. 4]. Denote by $A_{P}$ the right quotient ring of $A$ with respect to $\mathcal{C}_{A}(P)$. Suppose that $P$ is right localizable and contains no nonzero $H$-stable ideals of $A$. Is $M \otimes_{A} A_{P}$ then a projective $A_{P}$-module for every locally $A$-finite object $M \in{ }_{H} \mathcal{M}_{A}$ ? I can prove that this holds true under the assumption that all prime ideals of $A$ containing $P$ are maximal and $H$ is pointed with finitely many grouplike elements.

\section{REFERENCES}

[1] J., Bergen; M., Cohen; D., Fischman; Irreducible actions and faithful actions of Hopf algebras, Isr. J. Math. 72 (1990), 5-18. MR.1098978 (92g:16044)

[2] N., Bourbaki; Commutative Algebra, Springer (1989). MR1727221 (2001g:13001)

[3] E., Cline; B., Parshall; L., Scott; A Mackey imprimitivity theory for algebraic groups, Math. Z. 182 (1983), 447-471. MR0701363 (84j:14046)

[4] S., Dăscălescu; C., Năstăsescu; S., Raianu; Hopf Algebras, an Introduction, Pure and Applied Mathematics 235, Marcel Dekker (2000). MR.1786197 (2001j:16056) 
[5] M., Demazure; P., Gabriel; Groupes Algébriques I Masson (1970). MR0302656 (46:1800)

[6] Y., Doi; Cleft comodule algebras and Hopf modules, Comm. Algebra 12 (1984), 1155-1169. MR0738541 (86b:16006)

[7] Y., Doi; Algebras with total integrals, Comm. Algebra 13 (1985), 2137-2159. MR0801433 (87c:16013)

[8] Y., Doi; Unifying Hopf modules, J. Algebra 153 (1992), 373-385. MR1198206 (94c:16048)

[9] I., Doraiswamy; Projectivity of modules over rings with suitable group action, Comm. Algebra 10 (1982), 787-795. MR0651972 (83g:14024)

[10] D., Eisenbud; Commutative algebra with a view toward algebraic geometry, Graduate Texts in Math. 150 Springer (1995). MR 1322960 (97a:13001)

[11] F., Kasch; Moduln und Ringe, Teubner (1977). MR0429963 (55:2971)

[12] M., Koppinen; T., Neuvonen; An imprimitivity theorem for Hopf algebras, Math. Scand. 41 (1977), 193-198. MR0485966 (58:5758)

[13] M., Koppinen; Coideal subalgebras in Hopf algebras: Freeness, integrals, smash products, Comm. Algebra 21 (1993), 427-444. MR.1199682 (93j:16029)

[14] H.F., Kreimer; M., Takeuchi; Hopf algebras and Galois extensions of an algebra, Indiana Univ. Math. J. 30 (1981), 675-692. MR0625597 (83h:16015)

[15] V., Linchenko; Nilpotent subsets of Hopf module algebras, in "Groups, Rings, Lie and Hopf Algebras", Kluwer, 2003, pp. 121-127. MR 1995055 (2004g:16040)

[16] V., Linchenko; S., Montgomery; L.W., Small; Stable Jacobson's radicals and semiprime smash products, Bull. London Math. Soc. 37 (2005), 860-872. MR.2186719

[17] A., Masuoka; On Hopf algebras with cocommutative coradicals, J. Algebra 144 (1991), 451466. MR.1140616 (92k:16053)

[18] A., Masuoka; Freeness of Hopf algebras over coideal subalgebras, Comm. Algebra 20 (1992), 1353-1373. MR.1157912 (93d:16051)

[19] A., Masuoka; Coideal subalgebras in finite Hopf algebras, J. Algebra 163 (1994), 819-831. MR1265867 (95b:16038)

[20] A., Masuoka; Quotient theory of Hopf algebras, in "Advances in Hopf algebras", Lecture Notes Pure Appl. Math., Vol. 158, pp. 107-133, Marcel Dekker, 1994. MR1289423 (95e:16038)

[21] A., Masuoka; Y., Doi; Generalization of cleft comodule algebras, Comm. Algebra 20 (1992), 3703-3721. MR1191974 (93j:16030)

[22] A., Masuoka; D., Wigner; Faithful flatness of Hopf algebras, J. Algebra 170 (1994), 156-164. MR.1302835 (95i:16040)

[23] J.C., McConnell; J.C., Robson; Noncommutative Noetherian Rings, Wiley (1987). MR0934572 (89j:16023)

[24] S., Montgomery; Von Neumann finiteness of tensor products of algebras, Comm. Algebra 11 (1983), 595-610. MR0694775 (84k:16019)

[25] S., Montgomery; Hopf algebras and Their Actions on Rings, CBMS Regional Conference Series in Mathematics, 82 American Mathematical Society (1993). MR.1243637 (94i:16019)

[26] D., Mumford; Geometric Invariant Theory, Ergebnisse der Mathematik und ihrer Grenzgebiete, 34 Springer (1965). MR0214602 (35:5451)

[27] W.D., Nichols; M.B., Zoeller; A Hopf algebra freeness theorem, Amer. J. Math. 111 (1989), 381-385. MR0987762 (90c:16008)

[28] W.D., Nichols; M.B., Zoeller; Freeness of infinite dimensional Hopf algebras over grouplike subalgebras, Comm. Algebra 17 (1989), 413-424. MR0978483 (90c:16009)

[29] W.D., Nichols; M.B., Zoeller; Freeness of infinite dimensional Hopf algebras, Comm. Algebra 20 (1992), 1489-1492. MR0978483 (90c:16009)

[30] B., Parshall; L., Scott; An imprimitivity theorem for algebraic groups, Indag. Math. 42 (1980), 39-47. MR0565942 (81k:20059)

[31] D.E., Radford; Pointed Hopf algebras are free over Hopf subalgebras, J. Algebra 45 (1977), 266-273. MR0437582 (55:10506)

[32] D.E., Radford; Freeness (projectivity) criteria for Hopf algebras over Hopf subalgebras, J. Pure Appl. Algebra 11 (1977), 15-28. MR0476790 (57:16344)

[33] L.H., Rowen; Ring Theory, Vol. I, Academic Press (1988). MR0940245 (89h:16001)

[34] H.-J., Schneider; Principal homogeneous spaces for arbitrary Hopf algebras, Isr. J. Math. 72 (1990), 167-195. MR1098988 (92a:16047)

[35] H.-J., Schneider; Normal basis and transitivity of crossed products for Hopf algebras, J. Algebra 152 (1992), 289-312. MR.1194305 (93j:16032) 
[36] H.-J., Schneider; Some remarks on exact sequences of quantum groups, Comm. Algebra 21 (1993), 3337-3357. MR.1228767(94e:17026)

[37] S.M., Skryabin; An algebraic approach to the Lie algebras of Cartan type, Comm. Algebra 21 (1993), 1229-1336. MR1209931 (94a:17017)

[38] M.E., Sweedler; Hopf Algebras, Benjamin (1969). MR 0252485 (40:5705)

[39] M., Takeuchi; A correspondence between Hopf ideals and sub-Hopf algebras, Manuscripta Math. 7 (1972), 251-270. MR0321963 (48:328)

[40] M., Takeuchi; Formal schemes over fields, Comm. Algebra 5 (1977), 1483-1528. MR 0498540 $(58: 16645)$

[41] M., Takeuchi; Relative Hopf modules-equivalences and freeness criteria, J. Algebra 60 (1979), 452-471. MR0549940 (82m:16006)

[42] Y., Zhu; The dimension of irreducible modules for transitive module algebras, Comm. Algebra 29 (2001), 2877-2886. MR1849108 (2002i:16058)

Chebotarev Research Institute, Universitetskaya St. 17, 420008 Kazan, Russia

E-mail address: Serge.Skryabin@ksu.ru 